\title{
DISK EVOLUTION IN OB ASSOCIATIONS: DEEP SPITZER/IRAC OBSERVATIONS OF IC 1795
}

\author{
Veronica Roccatagliata $^{1,2}$, Jeroen Boumman ${ }^{1}$, Thomas Henning ${ }^{1}$, Mario Gennaro ${ }^{1}$, Eric Feigelson $^{3,4}$, \\ Jinyoung SEREna Kim ${ }^{5}$, Aurora Sicilia-Aguilar ${ }^{1}$, and Warrick A. Lawson ${ }^{6}$ \\ ${ }^{1}$ Max-Planck-Institut für Astronomie, Königstuhl 17, D-69117 Heidelberg, Germany \\ ${ }^{2}$ Space Telescope Science Institute, Baltimore, MD 21218, USA \\ ${ }^{3}$ Department of Astronomy and Astrophysics, Pennsylvania State University, University Park, PA 16802, USA \\ ${ }^{4}$ Center for Exoplanets and Habitable Worlds, 525 Davey Laboratory, Pennsylvania State University, University Park, PA 16802, USA \\ ${ }^{5}$ Steward Observatory, University of Arizona, 933 N. Cherry Ave. Tucson, AZ 85721-0065, USA \\ ${ }^{6}$ School of Physical, Environmental, and Mathematical Sciences, University of New South Wales, Australian Defence Force Academy, \\ Canberra, ACT 2600, Australia \\ Received 2010 September 30; accepted 2011 March 22; published 2011 May 13
}

\begin{abstract}
We present a deep Spitzer/Infrared Array Camera (IRAC) survey of the OB association IC 1795 carried out to investigate the evolution of protoplanetary disks in regions of massive star formation. Combining Spitzer/IRAC data with Chandra/Advanced CCD Imaging Spectrometer observations, we find 289 cluster members. An additional 340 sources with an infrared excess, but without X-ray counterpart, are classified as cluster member candidates. Both surveys are complete down to stellar masses of about $1 M_{\odot}$. We present pre-main-sequence isochrones computed for the first time in the Spitzer/IRAC colors. The age of the cluster, determined via the location of the Class III sources in the [3.6]-[4.5]/[3.6] color-magnitude diagram, is in the range of 3-5 Myr. As theoretically expected, we do not find any systematic variation in the spatial distribution of disks within $0.6 \mathrm{pc}$ of either O-type star in the association. However, the disk fraction in IC 1795 does depend on the stellar mass: sources with masses $>2 M_{\odot}$ have a disk fraction of $\sim 20 \%$, while lower mass objects $\left(2-0.8 M_{\odot}\right)$ have a disk fraction of $\sim 50 \%$. This implies that disks around massive stars have a shorter dissipation timescale.
\end{abstract}

Key words: ISM: individual objects (W3) - open clusters and associations: individual (W3 IC1795) protoplanetary disks - stars: formation - stars: pre-main sequence - X-rays: stars

Online-only material: color figures, machine-readable tables

\section{INTRODUCTION}

Circumstellar (CS) disks have been found around all types of objects, ranging from Herbig Ae/Be stars to brown dwarfs, sharing similar dust composition, although differences between the disks of low-mass stars/brown dwarfs, solar type stars, and more massive stars seem to exist in the evolution of both the dust and gas components (e.g., Henning \& Meeus 2009; Pascucci et al. 2009). Though the timescales of disk evolution and dispersion are not well constrained, $90 \%$ of the pre-main-sequence (PMS) systems appear to disperse the dust and gas within the planet formation zone within $\sim 10 \mathrm{Myr}$ (e.g., Mamajek et al. 2004; Bouwman et al. 2006; Fedele et al. 2010). Consequently, the formation of gas planets has to be completed within this timescale.

Although most solar-type and low-mass stars form in OB associations, disks studies around PMS stars deal mostly with regions of low-mass star formation in the Gould Belt (e.g., Hartmann et al. 2005; Winston et al. 2007; Hatchell et al. 2007; Sicilia-Aguilar et al. 2008; Evans et al. 2009) and nearby moving groups (e.g., Lyo et al. 2003; Sicilia-Aguilar et al. 2009; Meeus et al. 2009).

An exception is the Orion Nebular Cluster (e.g., Hillenbrand 1997; Hillenbrand et al. 1998), the nearest ( $410 \mathrm{pc}$; Jeffries 2007), young, dense, and rich cluster with the O6 star, $\theta^{1}$ Ori C, in the center. The disks are seen as dark silhouettes against the bright nebula background and they are called "proplyds" (e.g., O'dell \& Wong 1996). Studies of the disk fraction in Orion (e.g., Hillenbrand et al. 1998) reveal a higher number of disks within $0.5 \mathrm{pc}$ than at larger distances from the O6 star. This suggests that the central $\mathrm{O}$ star has had insufficient time to fully photoevaporate surrounding disks. However, Eisner et al. (2008) found that the percentage of disks more massive than $\sim 0.01 M_{\odot}$ is lower than in Taurus.

There is compelling observational evidence that these disks have been influenced by the ultraviolet radiation from $\theta^{1}$ Ori $\mathrm{C}$ (e.g., Johnstone et al. 1998; Clarke 2007; Gorti et al. 2009), in that the ionized gas surrounding these disks has a cometary shape (McCullough et al. 1995; Bally et al. 1998), their mass distribution is truncated at $0.03 M_{\odot}$, and the outer radii, in most of the cases, are smaller than $60 \mathrm{AU}$ (Mann \& Williams 2009). However, such a truncation in mass and radii distribution might be due to disk mass loss triggered by stellar encounters which are also responsible for the destruction of $10 \%-15 \%$ disks in the Trapezium cluster (e.g., Scally \& Clarke 2001; Olczak et al. 2006).

Investigations of disk dissipation processes induced by massive stars, such as photoevaporation and dynamical destruction, must be made in rich, crowded young clusters. To properly carry out such a study, a disk-unbiased census of cluster members and a robust tracer of disks are needed. Chandra X-ray surveys provide member lists based on PMS magnetic activity; these are particularly effective in locating the disk-free (Class III) stellar population (Feigelson et al. 2007). Spitzer mid-infrared surveys provide sensitive member lists based on infrared excess; these are particularly effective in locating the disky (Class $0-\mathrm{I}-\mathrm{II}$ ) stellar population. The X-ray and infrared approaches are complementary in other respects: the X-ray selected samples nicely discriminate young cluster members from older Galactic field stars that dominate infrared surveys, while the infrared samples detect the very low mass population with magnetic activity too faint to be detected in most X-ray exposures. 
The characterization of CS material in the inner part of the disks can be provided by sensitive Infrared Array Camera (IRAC) surveys, while a complete membership may be identified by an optical spectroscopy survey for low-mass members with spectral types $\mathrm{K}-\mathrm{M}$ (via the Li emission at $6708 \AA$, e.g., Jeffries et al. 2007; Mentuch et al. 2008) or accreting objects (via $\mathrm{H}_{\alpha}$ and UV-excess emission; Fedele et al. 2010; Sicilia-Aguilar et al. 2010; Fang et al. 2009). The solar-type mass population can be identified via an X-ray survey.

\subsection{Past Work}

This approach has been used by, e.g., Damiani et al. (2006), who combined a deep X-ray Chandra survey with an optical spectroscopic survey to study the population properties of the $5 \mathrm{Myr}$ old cluster NGC 2363, which hosts an O9.5I star. They found that only 5\%-9\% of the cluster members are still accreting.

Recently, Balog et al. (2007) studied disk evolution and the influence of the $\mathrm{O}$ stars in the high-mass star-forming region NGC 2244. Using IRAC observations, they found an overall disk fraction of $44.5 \%$, but that the fraction of sources with disks was lower $(\sim 27 \%)$ within 0.5 pc of the O stars.

Guarcello et al. $(2009,2007)$ analyzed the disk fraction in the high-mass OB association NGC 6611, which hosts 56 stars with spectral type earlier than B5. The cluster membership and the disk fraction were defined combining optical, near- and mid-infrared, and X-ray studies. The disk fraction for the $\mathrm{O}$ and $\mathrm{B}$ stars was found varying between $31 \%$ and $16 \%$ across a complete range of incident fluxes up to $800 \mathrm{erg} \mathrm{cm}^{-2} \mathrm{~s}^{-1}$. In a smaller region compared to the Guarcello et al. $(2009,2007)$ studies, Oliveira et al. (2005) computed a much higher disk fraction $(\sim 58 \%)$ probably due to the lower sensitivity of their survey.

Getman et al. (2009) studied a portion of the Cep OB3b cluster and the triggered population in the adjacent Cep B molecular cloud. Using a combined Chandra-Spitzer sample, they found that the disk fraction showed a spatial gradient from $75 \%$ in the cloud core to $25 \%$ in the unobscured cluster. They attributed the gradient to stellar ages rather than photoevaporation.

Hernández et al. (2008) computed the disk fraction in the 5 Myr old $\gamma$ Velorum cluster, with a central binary consisting of an 07.5 star and a Wolf-Rayet star. Only $5 \%$ of cluster members showed an infrared excess.

Despite these studies, the number of investigations concerning the presence of disks around stars in $\mathrm{OB}$ associations has been limited.

\subsection{This Work}

In this paper, we present the analysis of protoplanetary disks around young stars in the IC 1795 OB association. We obtained infrared imaging data of the IC 1795 OB association with the IRAC (Fazio et al. 2004) on board the Spitzer Space Telescope (Werner et al. 2004). To define the cluster membership of IC 1795 we carried out a deep survey with the Advanced color-color diagram (CCD) Imaging Spectrometer (ACIS) detector on board the Chandra X-ray Observatory (Weisskopf et al. 2002).

The IC 1795 cluster ionizes the diffuse H II region in the W3 giant molecular cloud complex. This region is located in the Perseus arm, which contains several spectacular regions of high- and low-mass star formation: W3-North, W3-Main, and W3-OH. IC 1795 hosts a bright O6.5V star, BD $+61^{\circ} 411$, originally spectrally classified by Mathys (1989), an O9.7I star and two B stars which have been spectrally classified in the optical by Oey et al. (2005). The cluster is assumed to lie at the same distance as W3-OH. From maser kinematics this has been accurately measured to be $2.0 \mathrm{kpc}$ (Xu et al. 2006; Hachisuka et al. 2006).

Oey et al. (2005) derived an approximate age of 3-5 Myr and they propose that this $\mathrm{OB}$ association, triggered by the neighboring W4 superbubble, is triggering new star formation in the young massive regions W3-North, W3-Main, and W3-OH.

The X-ray morphology of these regions suggests, however, that only the $\mathrm{W} 3-\mathrm{OH}$ structures are consistent with the collectand-collapse triggering process caused by shocks from the older IC 1795 cluster (Feigelson \& Townsley 2008). In the X-ray maps, the embedded W3-Main cluster does not show the elongated and patchy structure of a recently triggered star cluster, and instead it appears to have formed in an earlier episode.

Previous IRAC observations of the entire W3 region, presented by Ruch et al. (2007), revealed that a large fraction of Class II sources lie within the central cluster IC 1795. No analysis of the spatial distribution and disk fraction of the cluster has been performed so far. The photometric observations presented in this paper concentrate on the lightly obscured IC 1795 OB association, and they have one order of magnitude better sensitivity compared with the Ruch et al. (2007) observations. A new deeper X-ray Chandra survey of IC 1795 is also presented in this work to accurately define the cluster membership.

This paper is organized as follows: in Section 2 we present our Spitzer and Chandra observations, along with the data reduction. Results are shown in Section 3. Sections 4 and 5 include the analysis of the cluster (membership, age, infrared properties of the stars). In Section 6, we discuss our results in terms of disk evolution. Conclusions are drawn in Section 7.

\section{OBSERVATIONS AND DATA REDUCTION}

\subsection{IRAC Observations}

IC 1795 was observed in 2007 September $^{7}$ with all 4 channels centered at $3.6 \mu \mathrm{m}, 4.5 \mu \mathrm{m}, 5.8 \mu \mathrm{m}, 8.0 \mu \mathrm{m}$, respectively, using a $3 \times 4$ mosaic pattern, with pointings separated by $220^{\prime \prime}$ and aligned with the array axes. The resulting images provide full coverage over a $14^{\prime} \times 12^{\prime}$ area in all four IRAC channels.

The images have been obtained in high dynamical range mode in order to obtain unsaturated measurements for all observed cluster members. The maps are obtained in 144 cycles organized in 12-point dither patterns. In each channel, a short exposure of 144 frames of $0.4 \mathrm{~s}$ each and a long exposure of $10.4 \mathrm{~s}$ per 144 frames have been carried out. In each channel, the average short and long exposures over the entire mosaic are 58 and $1497 \mathrm{~s}$, respectively. The long and the short exposures are analyzed separately to avoid saturation of bright sources in the long exposures.

The raw data were processed and calibrated with the IRAC pipeline (version S16.1.0) and the Basic Calibrated Data (BCD) were downloaded from the Spitzer archive. ${ }^{8}$ The final mosaics were created using the MOPEX pipeline (version 18.2.2) ${ }^{9}$ : the software takes the individual BCD frames and combines them

\footnotetext{
7 Spitzer program ID 30726, PI: J. Bouwman.

8 http://archive.spitzer.caltech.edu/

9 http://irsa.ipac.caltech.edu/data/SPITZER/docs/dataanalysistools/tools/ mopex/mopexdownload
} 


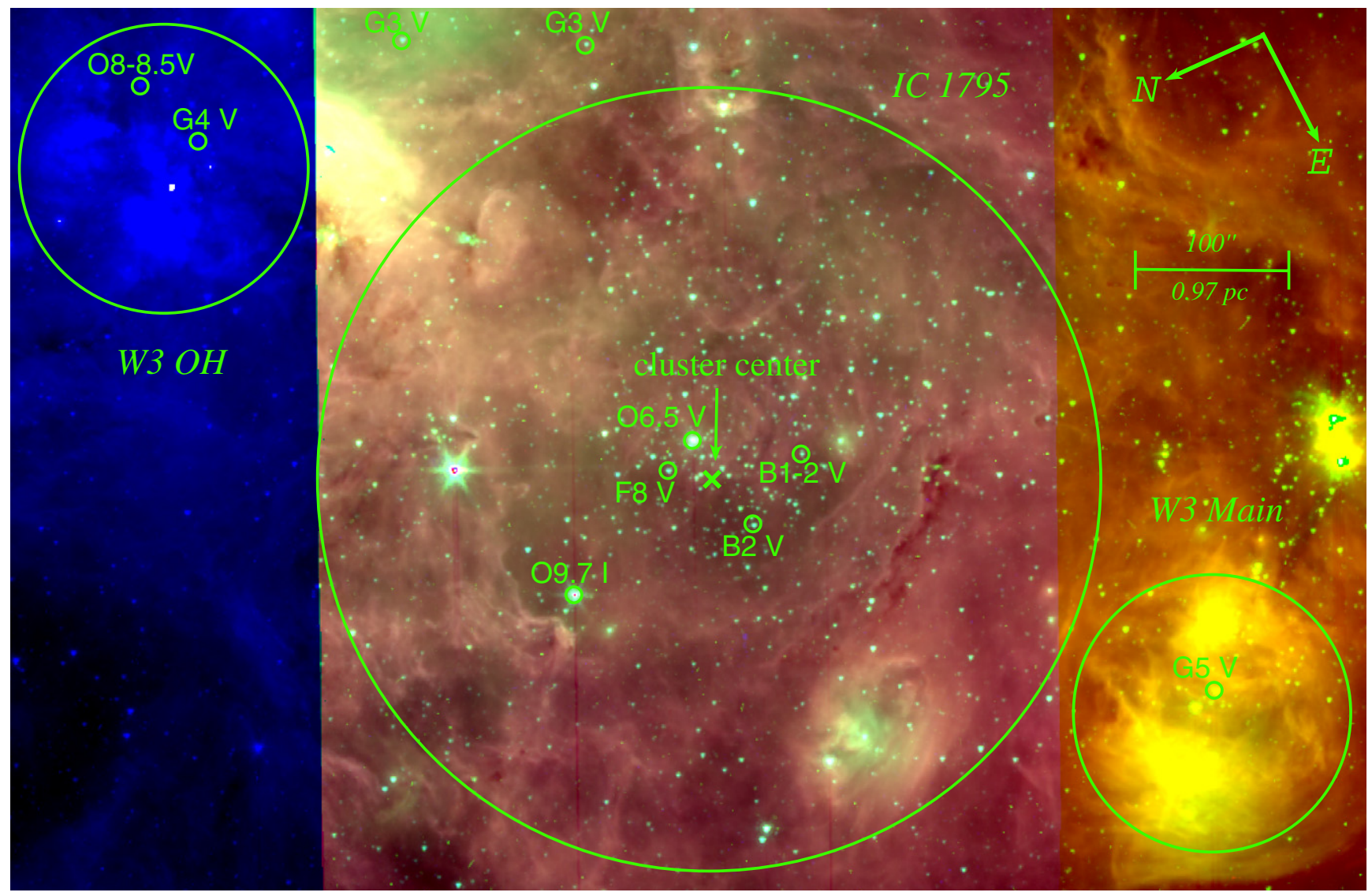

Figure 1. Combined image of the mosaics obtained with the long exposures of IRAC/ch1, IRAC/ch2, and IRAC/ch4. Highlighted are the edge and center of IC 1795 together with the positions of the high- and intermediate-mass stars in the region spectrally classified by Oey et al. (2005). W3-Main and W3-OH are not covered by all IRAC channels.

(A color version of this figure is available in the online journal.)

to create a mosaic of the observed region, removing cosmic rays and bad pixels from the single frames. The combined mosaic of the long exposures at $3.6 \mu \mathrm{m} / 4.5 \mu \mathrm{m} / 8.0 \mu \mathrm{m}$ of IC 1795 is shown in Figure 1, together with the positions of the center and the edge of the cluster. Due to the mosaic configuration, the exposures of ch1 and ch3 cover part of W3-Main, while ch2 and ch4 cover part of W3-OH.

\subsection{IRAC Photometry}

We used the APEX/MOPEX package to perform pointspread function (PSF) photometry for every detection. In particular, we used the APEX MultiFrame pipeline, where the detection of the point sources is done on the final mosaic in order to recover also the fainter objects, while the PSF photometry is carried out on each frame separately.

Over the cluster region, the background was highly variable. We estimated the background variability by considering six different regions of the cluster of $25 \times 25$ pixels each, corresponding to an area of $\sim 30^{\prime \prime} \times 30^{\prime \prime}$. The mean background flux at $3.6 \mu \mathrm{m}$ was about $0.07 \mathrm{Jy}$ with an average standard deviation of $0.05 \mathrm{Jy}$. At the edge of IC 1795, where it borders on the younger regions $\mathrm{W} 3-\mathrm{OH}$ on the southwest direction and on W3-Main on the northeast direction, the background increases up to $0.08 \mathrm{Jy}$ and $1.6 \mathrm{Jy}$ at 3.6 and $8 \mu \mathrm{m}$, respectively. Here, the background is most likely associated to the molecular clouds of the younger W3-OH and W3-Main regions. Over the cluster region it is instead associated with the polycyclic aromatic hydrocarbon emission which peaks at $3.3,6.3,7.7$, and $8.6 \mu \mathrm{m}$.
As the background was highly variable, PSF photometry was computed in a small area of $8 \times 8$ pixels and subtracted before performing PSF-photometry. Instead of PSF fitting, APEX uses the point-response function (PRF) fitting method. The PRF is the response of the detector array pixels to a point source and combines information on the PSF, the detector sampling, and the intra-pixel sensitivity variation. We used the standard PRFs provided in APEX. The point-source positional uncertainties and the flux uncertainty $\left(\Delta_{\mathrm{PRF}}\right)$ are computed from the covariance matrix calculated from the "best fit" to the data.

The calibration uncertainties (Reach et al. 2005) are dominated by the array-location-dependent photometric correction and the pixel phase effect which might introduce an uncertainty of up to $10 \%$ of the flux. However, since the pixel phase effect decreases as the square root of the number of dithers (12 in our case), we conservatively adopt a value of $5 \%$. The color correction and the absolute calibration error are on the order of a few percent. Overall the final flux calibration error $\left(\Delta_{\mathrm{cal}}\right)$ is smaller than $10 \%$ of the flux. The total flux uncertainty is

$$
\Delta_{\text {flux }}=\sqrt{\Delta_{\mathrm{PRF}}^{2}+\Delta_{\mathrm{cal}}^{2}} .
$$

Only sources with a signal-to-noise $(\mathrm{S} / \mathrm{N})$ flux measurement greater than 6 in each IRAC channel have been considered in our final catalog. The final photometric catalog was created, combining the results of the analysis of the short and long exposures separately. For objects detected in both exposures (and not saturated), we adopt the photometry measured in the 
long exposure. The detection limit of our IRAC survey in the $3.6 \mu \mathrm{m}$ band is $16.5 \mathrm{mag}$. However, we have cases where the high variability of the background did not enable us to detect all the sources with brightness between 15.5 and 16.5 mag at $3.6 \mu \mathrm{m}$. At $4.5 \mu \mathrm{m}$ we detected sources down to $16 \mathrm{mag}$, at $5.8 \mu \mathrm{m}$ and $13.3 \mathrm{mag}$, and at $8.0 \mu \mathrm{m}$ and $11.5 \mathrm{mag}$.

\subsection{ACIS Observations}

Our X-ray catalog was generated from a mosaic of Chandra observations consisting of the seven exposures of W3 presented by Feigelson \& Townsley (2008) and an additional exposure of IC 1795. This is a $50.0 \mathrm{ks}$ exposure obtained on 2007 December 4 centered on $\left(\alpha_{J 2000}, \delta_{J 2000}\right)=\left(02^{\mathrm{h}}: 26^{\mathrm{m}}: 33^{\mathrm{s}} \cdot 6,+62^{\circ}: 00^{\prime}: 35^{\prime \prime} .9\right)$ with the 4-CCD ACIS Imaging Array subtending $17^{\prime} \times 17^{\prime}$. The data were reduced using procedures implemented in the IDLbased Tools for ACIS Review and Analysis (TARA) and ACIS Extract software packages. The algorithms are described in Broos et al. (2010) and are available online. ${ }^{10}$ Their application to stellar populations in other star-forming regions include M17 (Broos et al. 2007), the Rosette Nebula (Wang et al. 2008, 2009), and NGC 6334 (Feigelson et al. 2009).

Summarizing briefly here, the Chandra satellite telemetry data are subject to various cleaning operations, and the image is aligned to the Two Micron All Sky Survey (2MASS)/Hipparcos astrometric frame. A superset of tentative sources is identified by a combination of wavelet-based source detection, peaks in image tiles with PSF removed by a maximum-likelihood image reconstruction, and visual inspection for close multiple sources. Photons are extracted from each tentative source in the broad soft, hard, and total X-ray bands, and photon counts are compared to the locally measured background. Local backgrounds are essential due to the different exposures from overlapping ACIS exposures. Source significance is evaluated statistically by the quantity $P_{B}$, the probability based on Poisson statistics that the observed source counts would be present given the observed local background rate.

Choosing a source significance limit $P_{B}<1 \%$ in at least one broad band, we find $2192 \mathrm{X}$-ray sources in the Chandra mosaic covering the W3-North, W3-Main, W3-OH, and IC 1795 regions. Source properties including $\mathrm{X}$-ray spectra, absorptions, luminosities, and variability will be presented in a forthcoming paper (L. K. Townsley et al. 2012, in preparation). For the present study, we consider only the X-ray source positions and error circles. Typical estimated $1 \sigma$ error circle radii are $0.2-0.5$ but can exceed $1^{\prime \prime}$ for sources far off-axis. Note that the Chandra positional errors are often considerably smaller than the IRAC positional errors shown in Figure 2 (panels (A) and (B)).

\subsection{Near-infrared Survey of IC 1795}

Near-infrared photometry of IC 1795 has been also analyzed. Near-infrared photometry in $J(1.25 \mu \mathrm{m}), H(1.65 \mu \mathrm{m})$, and $K s$ $(2.17 \mu \mathrm{m})$ bands is taken from the 2MASS (Skrutskie et al. 2006). ${ }^{11}$ The $3 \sigma$ limiting sensitivity of this survey is $17.1,16.4$, and 15.3 mag for the three bands, respectively.

\section{THE IRAC POINT SOURCE CATALOG}

\subsection{Positions of the IRAC Sources}

The final photometric catalogs have been filtered for position errors, rejecting sources with positional errors in R.A. and decl.

\footnotetext{
$10 \mathrm{http} / / /$ www.astro.psu.edu/xray/docs/TARA

11 Only good photometry denoted by the flag $A A A$ has been considered.
}

larger than 3.'6 (two times the FWHM of the PSF in all IRAC channels). The distribution of source positions as a function of their error shows that sources with a larger position error are the fainter targets with a larger uncertainty in the flux (see Figure 2 for channel 1).

The histograms of the source positions in panels (C) and (D) of Figure 2 show a bimodal structure which reflects the distribution of stars in IC 1795 and W3-Main (ch1-ch3), and of stars in IC 1795 and W3-OH (ch2-ch4). From the peak of the histogram centered on IC 1795 , the center of the cluster is found to be $\left(\alpha_{J 2000}, \delta_{J 2000}\right)=\left(02^{\mathrm{h}}: 26^{\mathrm{m}}: 39^{\mathrm{s}},+62^{\circ}: 00^{\prime}: 41^{\prime \prime}\right)$. This is $\sim 4^{\prime}$ southeast of the cluster center chosen by Oey et al. (2005).

In panels (A) and (B) of Figure 3, the density distribution of all infrared sources $\left(D_{\text {star }}\right)$ is analyzed. $D_{\text {star }}$ is computed as the total number of sources in a ring $(N)$, defined between two consecutive radii from the cluster center, divided by the ring area $\left(A_{\text {ring }}\right)$. The annuli start at $60^{\prime \prime}$ from the center of the cluster and end at $330^{\prime \prime}$. The width of each annulus is $30^{\prime \prime}$. The errors in the density distribution are computed as $\sqrt{N} / A_{\text {ring. }}$. The density distribution of sources detected in at least one IRAC channel has been analyzed as a function of the distance from the cluster center (panel (A) of Figure 5). $D_{\text {star }}$ peaks at $90^{\prime \prime}$ from the center of the cluster and smoothly declines until 330" with the exception of a second minor peak at $~ 270^{\prime \prime}$ (panel (B) of Figure 3). The density distribution is consistent with a cluster extension of $\sim 3.2 \mathrm{pc}$. The first peak in density distribution at $\sim 0.9 \mathrm{pc}$ from the cluster center originates from a small clump of stars in the northwest direction and by an asymmetric distribution of the stars in IC 1795.

\subsection{Photometry of the IRAC Sources}

The four Spitzer channels were matched using the IDL procedure match_xy.pro in the IDL-based TARA package (see footnote 10; Broos et al. 2010). The source matching is based on agreement of positions assuming that positional errors are two-dimensional Gaussians at the $99 \%$ confidence level.

The total number of point sources detected decreases as the IRAC wavelength increases due to a decrease in sensitivity. In particular, within $330^{\prime \prime}$ from the cluster center, we detect $918,841,303$, and 243 sources at $3.6 \mu \mathrm{m}, 4.5 \mu \mathrm{m}, 5.8 \mu \mathrm{m}$, and $8.0 \mu \mathrm{m}$, respectively. 143 objects were detected in all four IRAC channels.

The CCD of the sources detected in all four IRAC channels and the color-magnitude diagram (CMD) of the sources detected at $3.6 \mu \mathrm{m}$ and $4.5 \mu \mathrm{m}$ (the most sensitive) are shown in panels (C) and (D) of Figure 3 (including the sources detected in all four bands). Megeath et al. (2004) and Allen et al. (2004) proposed a conversion scheme from IRAC colors to source classification (Class 0 , I, II, III sources), which has been later improved by Gutermuth et al. (2008, 2009).

The results of this classification for our sources are summarized in Table 1. Using the CMD in panel (C) of Figure 3, we distinguish between sources with infrared excess (characteristic of CS disks around young star; [3.6]-[4.5] $\geqslant 0.2 \mathrm{mag}$ ) and "photospheric sources" without infrared excess ([3.6]-[4.5] $<0.2 \mathrm{mag})$. The excess-threshold adopted here ([3.6]-[4.5] $=0.2 \mathrm{mag})$ takes into account two factors: (1) model isochrones computed in the IRAC bands do not predict [3.6]-[4.5] colors exceeding $0.1 \mathrm{mag}$; see Section 5.1 and Appendix A for details and (2) the maximum uncertainty of our photometry $(0.07 \mathrm{mag})$. Out of 592 sources detected at $3.6 \mu \mathrm{m}$ and $4.5 \mu \mathrm{m}, 327$ show an infrared excess and 265 are classified 


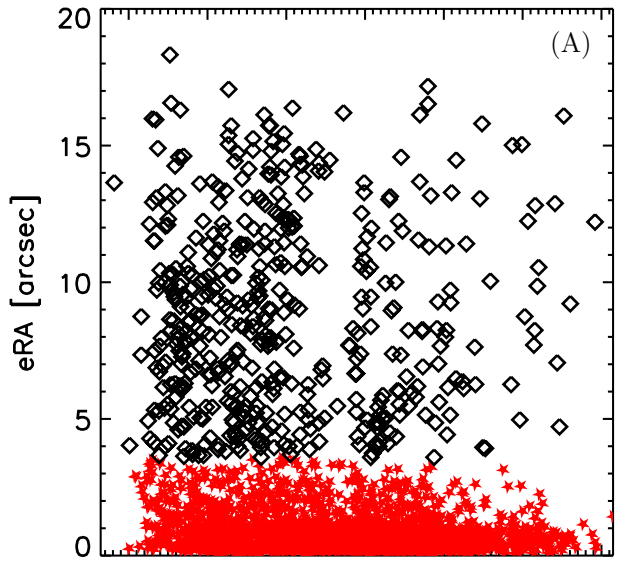

$\begin{array}{lllllll}36.3 & 36.4 & 36.5 & 36.6 & 36.7 & 36.8 & 36.9\end{array}$ RA [deg]

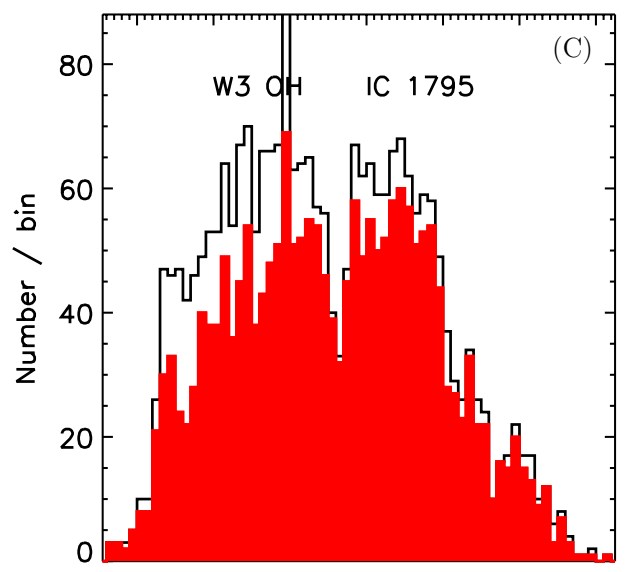

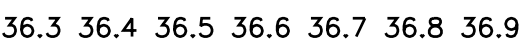
RA [degree]

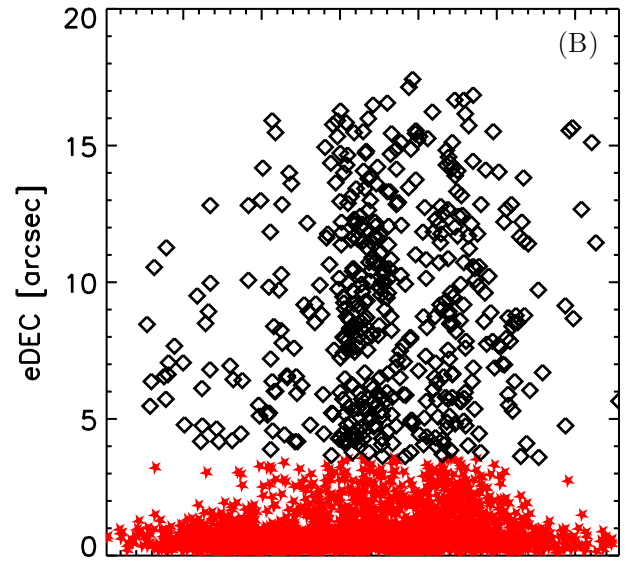

61.9562 .0062 .0562 .1062 .1562 .20 DEC [deg]

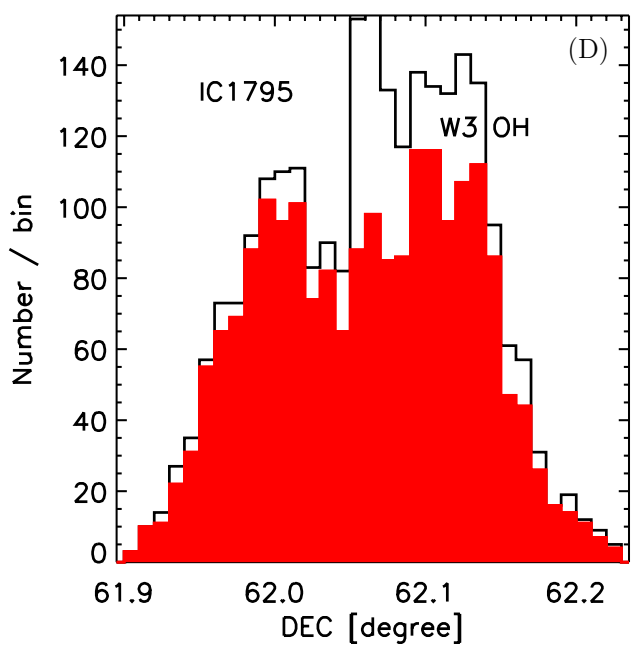

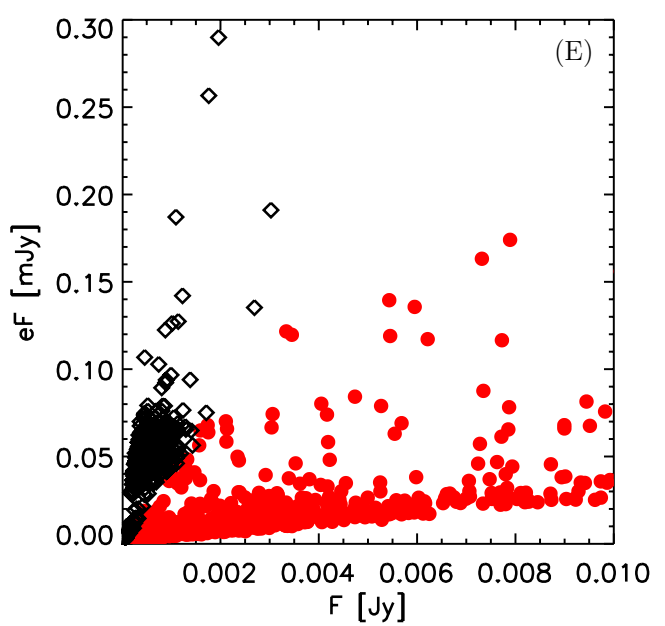

Figure 2. (A and B) R.A. and decl. errors vs. R.A. and decl. coordinates of all the sources detected in IRAC/ch1. The empty diamonds represent sources with coordinate errors larger than 3".6. Sources with positional errors less than 3.'6 are highlighted with filled red stars. (C and D) Black empty histograms represent the R.A. and decl. coordinates of all sources shown in panels (A) and (B) with empty diamonds. The red filled histograms represent R.A. and decl. coordinates of sources of our final lists (filled red stars in panels (A) and (B)). (E) Flux error vs. the flux of the sources shown in the upper panels. The red filled stars represent the sources of our final lists selected with position errors in R.A. and decl. $\leqslant 3$ ". 6 . Positional errors $>3$ "' 6 (black empty diamonds) correspond to the fainter sources with larger positional errors.

(A color version of this figure is available in the online journal.)

as "photospheric" sources. In the following sections, we will call "infrared sources" objects detected at least in one Spitzer channel, and "excess sources" objects with [3.6]-[4.5] $\geqslant 0.2$ mag.

Out of 66 Class I and Class II sources previously classified using the definitions of Megeath et al. (2004) and Allen et al.
(2004), all sources have a [3.6]-[4.5] $\geqslant 0.2$. Out of 57 ClassI_II sources, $60 \%$ have $[3.6]-[4.5] \geqslant 0.2$. This ensures the reliability of the new scheme adopted to determine a Class I and Class II sources. It is important to note that the effect of the visual extinction is negligible at these wavelengths: following the 

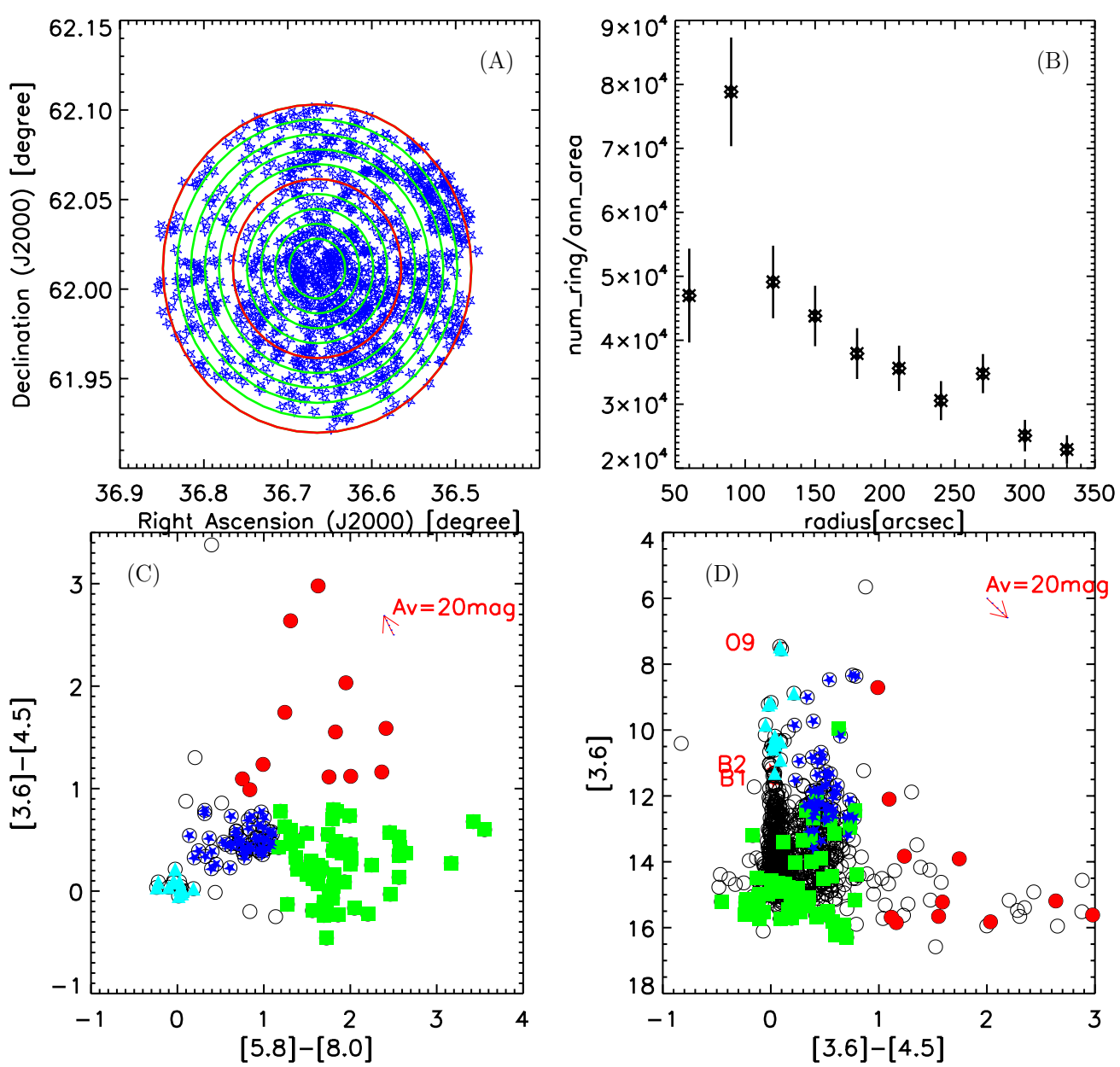

Figure 3. Spatial density distribution of all IR sources (A) and their spatial density distribution as a function of the distance from the center of the cluster (B). The green circles represent radii from $60^{\prime \prime}$ from the center of the cluster to $330^{\prime \prime}$ with $30^{\prime \prime}$ spacing. (C) Color-color diagram (CCD) of the IRAC/Spitzer sources detected within 330" from the center of the cluster. The different symbols and colors represent the disk classification from Megeath et al. (2004) and Allen et al. (2004): filled (cyan) triangles: photosphere/Class III; filled (green) squares: Class I/II; filled (blue) stars: Class II; filled (red) circles: Class O/I; (D) Color-magnitude diagram (CMD) of all the sources identified at $3.6 \mu \mathrm{m}$ and $4.5 \mu \mathrm{m}$. The different symbols represent the sources detected in four IRAC channels classified in the CCD on the right. Empty (black) circles: sources with magnitudes that do not follow the previous classification. The arrows represent an extinction $A_{V}=20 \mathrm{mag}$.

(A color version of this figure is available in the online journal.)

extinction relations of Cardelli et al. (1989), $A_{\mathrm{V}}=2$ mag (typical of a cluster at $2 \mathrm{kpc}$ ) corresponds to $A_{[3.6]}=5.9 \times 10^{-2} \mathrm{mag}$.

Ruch et al. (2007) analyzed the IRAC observations of the entire W3 region available in the Spitzer archive (GTO PID 127) and obtained with a total exposure time of $63.6 \mathrm{~s}$ in all IRAC channels. This corresponds to our short exposure, and it is almost one order of magnitude shorter than our long exposure time. They performed PSF photometry using a version of DAOPHOT modified by the Galactic Legacy Infrared Mid-Plane Survey Extraordinaire (GLIMPSE) team.

Within $330^{\prime \prime}$ from the center of the cluster, they identified 77 sources visible in all four IRAC channels. With our deeper infrared survey we detected 143 sources. There are however some differences between the two surveys: at $3.6 \mu \mathrm{m}$ and $4.5 \mu \mathrm{m}$ we do confirm 76/77 sources previously identified by Ruch et al. (2007), while 1 source has a position error larger than 3.'6 and it is not included in our analysis. At $5.8 \mu \mathrm{m}$ we confirmed the detection of 60 sources. Of the remaining 17 sources, 3 have position errors larger than 3.'6, while 14 have not been detected by the PRF fitting method performed in APEX. At $8.0 \mu \mathrm{m}$ we identified 32 sources. Of the remaining 45 sources, 26 have position errors larger than $3^{\prime \prime} 6$, while 19 have not been detected with APEX. We inspected all the non-matched detections (14 at
$5.8 \mu \mathrm{m}$ and 19 at $8.0 \mu \mathrm{m}$ ) in our deeper survey. In all cases we find that the local background is highly variable compared to the source brightness and for this reason we did not detect any point source with our PRF fitting method.

In summary we confirm 76 sources out of 77 sources detected by Ruch et al. (2007) in the ch1 of IRAC and 32 sources in all four IRAC channels. In addition to these sources we identified 111 new sources in all four channels.

\subsection{Extragalactic Contamination of the IR Catalog}

The extragalactic contamination of the infrared source catalog is estimated using the $3.6 \mu \mathrm{m}$ cumulative extragalactic counts from the IRAC/GOODS sample together with the incompleteness correction from Franceschini et al. (2006). About 10 galaxies brighter than $1 \mathrm{mJy}(\sim 13.6 \mathrm{mag})$ at $3.6 \mu \mathrm{m}$ are expected in an area of $1^{\circ} \times 1^{\circ}$. The number of galaxies increases to about one order of magnitude up to $\sim 300$ sources with fluxes higher than $0.1 \mathrm{mJy}$. Scaling these values for the IC 1795 area of about $0.26 \times 0.26$ the contamination of extragalactic sources is negligible (about 2.6 sources) down to $13.6 \mathrm{mag}$. In the $13.6-15.5 \mathrm{mag}$ range, about 70 sources $(\sim 8 \%$ 
Table 1

Total Number of Spitzer and Chandra Sources Detected within $330^{\prime \prime}$ from the Center of the Cluster

\begin{tabular}{|c|c|}
\hline & Number of Sources \\
\hline \multicolumn{2}{|c|}{ Spitzer observations } \\
\hline$\overline{\mathrm{ch} 1}$ & 924 \\
\hline $\operatorname{ch} 2$ & 845 \\
\hline $\operatorname{ch} 3$ & 309 \\
\hline $\operatorname{ch} 4$ & 248 \\
\hline $\operatorname{ch} 1+\operatorname{ch} 2$ & 841 \\
\hline With excess & 367 \\
\hline Without excess & 474 \\
\hline $\operatorname{ch} 1+\operatorname{ch} 2+\operatorname{ch} 3+\operatorname{ch} 4$ & 145 \\
\hline Class I & 12 \\
\hline Class I/II & 57 \\
\hline Class II & 54 \\
\hline Class III/phot & 13 \\
\hline not class & 9 \\
\hline \multicolumn{2}{|c|}{ Chandra + Spitzer observations } \\
\hline X-ray & 413 \\
\hline $\mathrm{X}-\mathrm{ray}+\mathrm{IR}^{\mathrm{a}}$ & 277 \\
\hline$X-$ ray $+\operatorname{ch} 1+\operatorname{ch} 2$ & 244 \\
\hline With excess & 116 \\
\hline Without excess & 128 \\
\hline $\mathrm{X}-\mathrm{ray}+\operatorname{ch} 1+\operatorname{ch} 2+\operatorname{ch} 3+\operatorname{ch} 4$ & 54 \\
\hline Class I & 1 \\
\hline Class I/II & 6 \\
\hline Class II & 41 \\
\hline Class III/phot & 4 \\
\hline not class & 2 \\
\hline X-ray without IR & 124 \\
\hline IR without X-ray & 665 \\
\hline $\operatorname{ch} 1+\operatorname{ch} 2$ without X-ray & 593 \\
\hline with excess & 248 \\
\hline without excess & 345 \\
\hline
\end{tabular}

Note. ${ }^{a}$ Nine X-ray sources have multiple infrared counterparts and they were counted as a single source.

of the total sources detected at $3.6 \mu \mathrm{m}$ ) might contaminate the IR source catalog of IC 1795 .

\section{CLUSTER MEMBERSHIP}

As outlined in the introduction, a full understanding of protoplanetary disk evolution in rich stellar clusters requires a cluster membership sample identified in a disk-unbiased way. Many photometric surveys of protoplanetary disks have been pursued with Spitzer, but Spitzer data alone suffer an important limitation: despite the excellent characterization of disks, sensitive IRAC surveys have biased the ratio of disked systems to non-disked systems in favor of the disk sources.

Optical spectroscopy is widely used to identify cluster members in star-forming regions. This is mainly based on two spectral features: the presence of $\mathrm{H} \alpha$ emission at $6563 \AA$ and strong absorption of the Li line at $6708 \AA$. These diagnostics are powerful tools for identifying young high accreting objects $(\mathrm{H} \alpha)$, or young low-mass stars with spectral type of $\mathrm{K}-\mathrm{M}$ (Li). However, this method is not effective at identifying earlier spectral type members: G-type stars, e.g., do not show a strong, age dependent Li I absorption and if they are non-accreting they show only tiny or zero $\mathrm{H} \alpha$ emission. An alternative, robust approach to study the complete population of a young stellar cluster is to combine sensitive X-ray and infrared observations (e.g., Mercer et al. 2009; Stelzer \& Scholz 2009; Wang et al. 2008; Getman et al. 2009).
Sensitive X-ray surveys are effective in identifying PMS stellar populations due to their enhanced magnetic flaring compared to older stars (e.g., Feigelson et al. 2007). Fluxlimited X-ray samples have the advantage of selecting young stars both with and without disks, but have the disadvantage of missing stars with fainter bolometric luminosities and lower masses. X-ray selected samples are complementary to Spitzer samples because they suffer less contamination from Galactic field stars or from diffuse nebular emission than infrared surveys. Getman et al. (2009) provide details on the relationships between stellar samples selected in Chandra and Spitzer surveys of a star-forming region. The high-spatial resolution telescope on the Chandra X-ray Observatory is essential to resolve crowded regions such as the IC 1795 and the W3 complex.

For IC 1795 we classified as cluster members sources detected in both, our infrared and X-ray, surveys, recognizing that extragalactic contamination may still be present (see the discussion in Section 4.2). We also considered as potential members sources detected by Spitzer which show an infrared excess at $4.5 \mu \mathrm{m}$ but lack an X-ray counterpart. This approach is fundamental when studying distant young clusters $(>1 \mathrm{kpc})$ that can be strongly contaminated by background and foreground stars.

\subsection{Cluster Membership Based on X-ray Detection}

Chandra source locations are matched to IRAC source locations using the IDL procedure match_xy.pro in the TARA package (see footnote 10; Broos et al. 2010). Associations between Chandra and Spitzer sources are assumed to be real when the probability that the X-ray and infrared sources are coincident exceeds $99 \%$, assuming bivariate Gaussian error distributions for the two source positions. Within 330" from the cluster center, we find 280 associations between X-ray and infrared sources, and 9 cases where a single IRAC source has two or more possible Chandra counterparts. The Chandra-Spitzer matched positions are listed in Table 2. The corresponding optical, 2MASS, and IRAC photometry is compiled in Table 3.

We analyzed the distribution of infrared sources with X-ray counterpart as a function of the distance from the cluster center (panels (A) and (B) of Figure 5). The distribution peaks at $\sim 90^{\prime \prime}$ from the center, declines down to $200^{\prime \prime}$, and then remains constant to $330^{\prime \prime}$ (except for a second peak at $\sim 270^{\prime \prime}$ ). The positions of these two peaks are also found in the distribution of infrared source positions (Section 3.1, panel (B) of Figure 3).

The number of X-ray sources alone, with and without infrared counterpart, and infrared sources (e.g., detected at least in one Spitzer channel) without X-ray counterpart are summarized in Table 1. The final catalog of the IC 1795 cluster members is in Table 3.

\subsection{Contaminants in the $X$-ray Catalog}

The sensitivity limit to X-ray sources in the IC 1795 cluster using the ACIS Extract methods is approximately $L_{x} \sim 5 \times 10^{29}$ $\mathrm{erg} \mathrm{s}^{-1}$ in the Chandra total band, $0.5-8 \mathrm{keV}$, assuming moderate absorption around $N_{\mathrm{H}} \sim 10^{21}-10^{22} \mathrm{~cm}^{-2}\left(A_{V} \sim 0.5-5 \mathrm{mag}\right)$ and source spectra typical of PMS stars. This is not a wellestablished value due to differences in source spectra, spatial variations in extinction, loss of sensitivity off-axis due to degradation of the Chandra point-spread function, and gain of sensitivity in some regions due to overlapping exposures in the W3 ACIS mosaic. In this paper, we use only the X-ray positions. A more detailed analysis of the X-ray observations 
Table 2

Spitzer/IRAC Sources with Chandra Counterparts

\begin{tabular}{ccccccc}
\hline \hline $\begin{array}{c}\text { ID } \\
\text { IRAC- }\end{array}$ & $\begin{array}{c}\text { R.A. } \\
(\mathrm{deg})\end{array}$ & $\begin{array}{c}\text { R.A. Error } \\
10^{-4}(\mathrm{deg})\end{array}$ & $\begin{array}{c}\mathrm{DE} \\
(\mathrm{deg})\end{array}$ & $\begin{array}{c}\text { Decl. Error } \\
10^{-4}(\mathrm{deg})\end{array}$ & $\begin{array}{c}\text { Infrared } \\
\text { Excess }\end{array}$ & $\begin{array}{c}\text { Disk } \\
\text { Classification }\end{array}$ \\
\hline $02255380+6201164$ & 36.474186 & 1.13 & 62.021217 & 1.17 & $\checkmark$ & \\
$02255585+6201477$ & 36.482689 & 2.54 & 62.029911 & 2.38 & - & \\
$02255585+6201477$ & 36.482689 & 2.54 & 62.029911 & 2.38 & - & \\
$02255717+6159399^{\mathrm{a}}$ & 36.488209 & 4.14 & 61.994419 & 4.24 & $\checkmark$ & \\
$02255780+6201287$ & 36.490833 & 2.72 & 62.024651 & 2.44 & $\sim$ & Class II \\
$02255891+6158121$ & 36.495461 & 0.48 & 61.970020 & 0.50 & $\checkmark$ & \\
$02255931+6202527$ & 36.497120 & 1.88 & 62.047962 & 1.84 & $\checkmark$ & \\
$02255900+6203133$ & 36.495831 & 4.03 & 62.053684 & 4.17 & $\checkmark$ & \\
$02255940+6159089$ & 36.497513 & 1.54 & 61.985809 & 1.62 & - & \\
$02255945+6201413$ & 36.497692 & 1.00 & 62.028152 & 1.03 & $\checkmark$ & \\
\hline
\end{tabular}

Notes. Notation for the infrared excess: " $\checkmark$ " $=[3.6]-[4.5]>0.2 \mathrm{mag}$; “-" $=[3.6]-[4.5]<0.1 \mathrm{mag}$; “ " $=0.1<[3.6]-[4.5]<$ $0.2 \mathrm{mag}$.

${ }^{a}$ Source position in IRAC/channel 2.

${ }^{\mathrm{b}}$ Source position in IRAC/channel 3.

${ }^{c}$ The counterparts at different wavelengths were found using a different matching procedure (see Section 4.1 for details).

d IRAC source with two X-ray counterparts ("X binary").

${ }^{\mathrm{e}}$ IRAC source with three X-ray counterparts.

(This table is available in its entirety in a machine-readable form in the online journal. A portion is shown here for guidance regarding its form and content.)

Table 3

Optical, Near-infrared, and IRAC/Spitzer Fluxes of the IRAC/Spitzer Sources with X-ray Counterpart

\begin{tabular}{|c|c|c|c|c|c|c|c|c|c|}
\hline $\begin{array}{c}\text { ID } \\
\text { IRAC- }\end{array}$ & $\begin{array}{c}\mathrm{FV} \\
(\mathrm{mJy})\end{array}$ & $\begin{array}{c}\text { FI } \\
(\mathrm{mJy})\end{array}$ & $\begin{array}{c}\text { FJ } \\
(\mathrm{mJy})\end{array}$ & $\begin{array}{c}\text { FH } \\
(\mathrm{mJy})\end{array}$ & $\begin{array}{c}\text { FK } \\
(\mathrm{mJy})\end{array}$ & $\begin{array}{l}\text { F3.6 } \\
\text { (mJy) }\end{array}$ & $\begin{array}{l}\mathrm{F} 4.5 \\
(\mathrm{mJy})\end{array}$ & $\begin{array}{c}\text { F5.8 } \\
\text { (mJy) }\end{array}$ & $\begin{array}{l}\text { F8.0 } \\
\text { (mJy) }\end{array}$ \\
\hline $02255380+6201164$ & $\ldots$ & $\ldots$. & $\ldots$. & $\ldots$ & $\ldots$ & $1.3026 \pm 0.0073$ & $1.3182 \pm 0.0088$ & $\ldots$ & $\ldots$ \\
\hline $02255585+6201477$ & $\ldots$ & $\ldots$ & $\ldots$ & $\ldots$ & $\ldots$ & $0.4586 \pm 0.0048$ & $0.3066 \pm 0.0055$ & $\ldots$ & $\ldots$ \\
\hline $02255585+6201477$ & $\ldots$. & $\ldots$ & $\ldots$ & $\ldots$ & $\ldots$ & $0.4586 \pm 0.0048$ & $0.3066 \pm 0.0055$ & $\ldots$ & $\ldots$ \\
\hline $02255717+6159399$ & 0.0073 & 0.0707 & $\ldots$. & $\ldots$ & $\ldots$ & $\ldots$ & $0.1901 \pm 0.0033$ & $\ldots$ & $\ldots$ \\
\hline $02255780+6201287$ & $\ldots$. & $\ldots$. & $\ldots$. & $\ldots$. & $\ldots$. & $0.4427 \pm 0.0048$ & $0.3130 \pm 0.0054$ & $\ldots$ & $\ldots$ \\
\hline $02255891+6158121$ & 0.0616 & 0.7341 & 4.0410 & 7.8397 & 8.2401 & $8.2684 \pm 0.0247$ & $8.7915 \pm 0.0271$ & $9.0294 \pm 0.2101$ & $9.3836 \pm 0.0890$ \\
\hline $02255931+6202527$ & $\ldots$. & $\ldots$. & $\ldots$. & $\ldots$. & $\ldots$. & $0.7768 \pm 0.0065$ & $0.6863 \pm 0.0088$ & $2.1174 \pm 0.0455$ & $\ldots$ \\
\hline $02255900+6203133$ & $\ldots$. & $\ldots$. & $\ldots$. & $\ldots$. & $\ldots$ & $0.4109 \pm 0.0073$ & $0.6331 \pm 0.0088$ & $\ldots$ & $\ldots$. \\
\hline $02255940+6159089$ & 0.0582 & 0.4375 & 1.0532 & 1.5357 & 1.1693 & $0.7298 \pm 0.0054$ & $0.3884 \pm 0.0039$ & $1.7319 \pm 0.0251$ & $2.0907 \pm 0.0523$ \\
\hline $02255945+6201413$ & $\ldots$. & $\ldots$. & $\ldots$. & $\ldots$. & $\ldots$. & $2.3144 \pm 0.0117$ & $2.4546 \pm 0.0133$ & $2.5519 \pm 0.0399$ & $\ldots$ \\
\hline
\end{tabular}

Notes. The near-infrared fluxes are from the 2MASS catalog (Skrutskie et al. 2006), selecting only sources flagged "AAA.” The optical fluxes are from J. José et al. (2011, in preparation). The IRAC flux errors refer to the covariance matrix calculated from the "best fit" of the PRF to the data and do not include calibration uncertainty $(\sim 10 \%$ of the flux $)$.

(This table is available in its entirety in a machine-readable form in the online journal. A portion is shown here for guidance regarding its form and content.)

will be presented in a forthcoming paper (L. K. Townsley et al. 2012, in preparation). We estimate the completeness limit to be around $L_{x} \sim 1 \times 10^{30} \mathrm{erg} \mathrm{s}^{-1}$. Based on the well-established empirical correlation between X-ray luminosity and stellar mass (Telleschi et al. 2007), this X-ray limit corresponds to a mass completeness limit of about $1 M_{\odot}$, although a considerable number of lower mass stars will be included in the Chandra sample.

A fraction of the X-ray sources should be contaminants, uniformly distributed, unrelated to the IC 1795 cluster (see discussion in Getman et al. 2006). About 30-40 faint and heavily absorbed X-ray sources in a $100 \mathrm{arcmin}^{2}$ region (which is of the order of the cluster size) will be background extragalactic objects, mainly quasars and AGNs, seen through the molecular material in the W3 complex and Galaxy along the line of sight. At least 20-30 lightly absorbed X-ray sources in a $100 \mathrm{arcmin}^{2}$ region should be foreground Galactic field stars, and $\sim 10$ sources should be background stars. The value depends on the amount of recent star formation along the line of sight to the W3 complex.
We expect that most of these contaminants, except for foreground stars, will not have an infrared counterpart and so will not contribute to our source list. These extragalactic and Galactic contaminants in the X-ray sample will exhibit a random spatial distribution, as is clearly shown for X-ray sources without infrared counterpart in panels $(\mathrm{E})$ and $(\mathrm{F})$ of Figure 5. However, a few quasars can have IRAC colors and X-ray fluxes similar to CTTS (e.g., Richards et al. 2009). This last source of contamination can only be removed via optical spectroscopy.

\subsection{Cluster Member IR Properties}

In Section 4.1, the cluster members have been defined as an infrared source with a X-ray counterpart. In panels (A) and (B) of Figure 4 the $C C D$ and CMD of the cluster members (i.e., infrared sources with X-ray counterpart) detected within $330^{\prime \prime}$ from the center of the cluster are shown. We distinguish between cluster members with infrared excess ([3.6]-[4.5] $\geqslant 0.2)$ and "photospheric" sources without infrared excess ([3.6]-[4.5] $<0.2)$.

In panel (A) of Figure 4, the 145 sources detected in all IRAC channels are shown. In Table 1 , we summarized the classification 

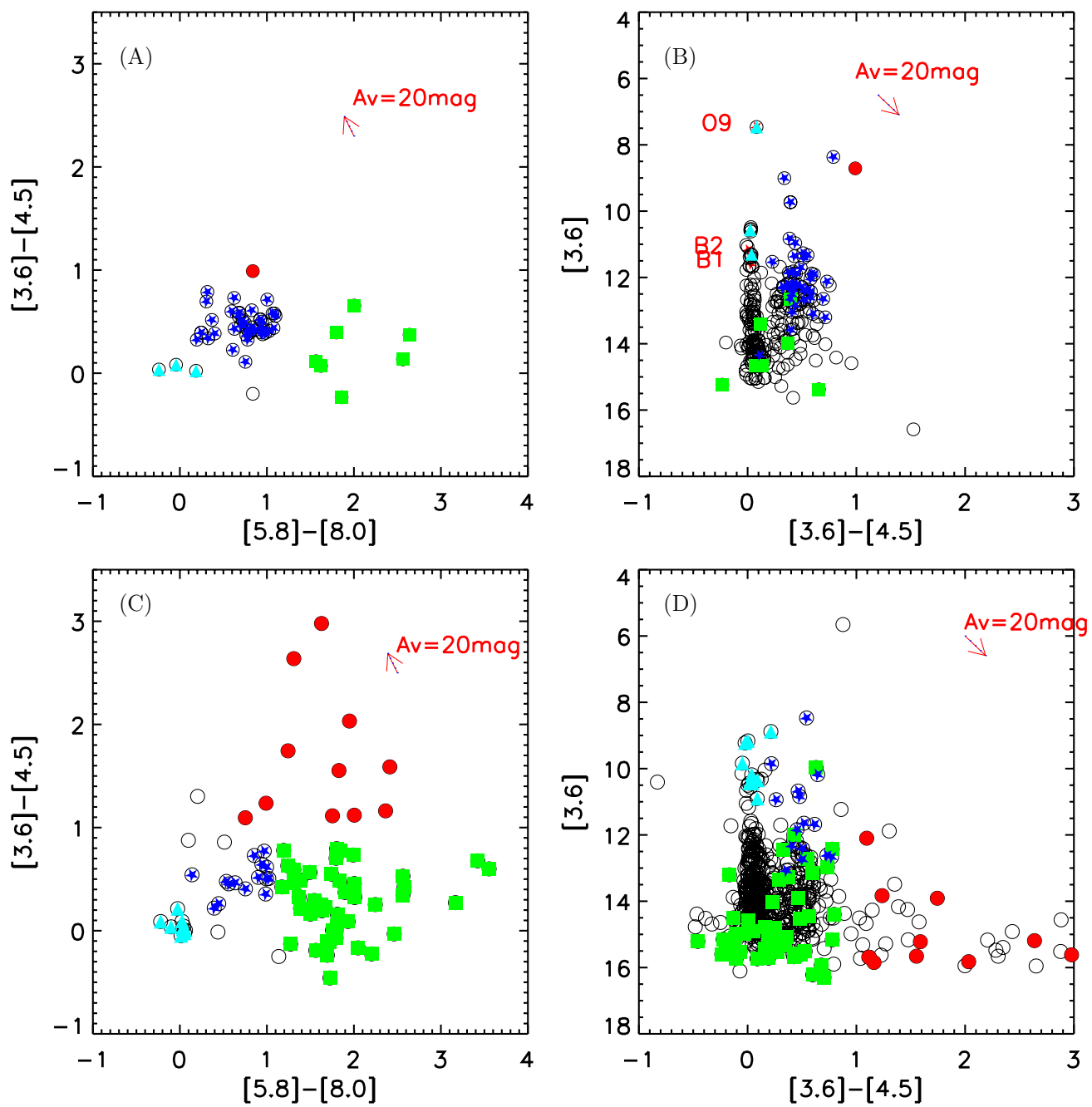

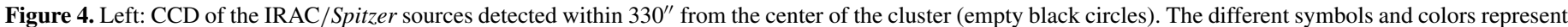

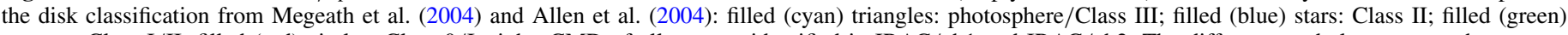

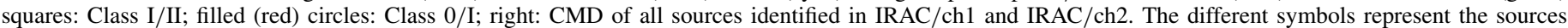

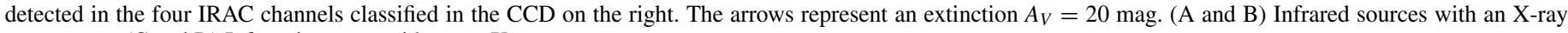
counterpart. (C and D) Infrared sources without an X-ray counterpart.

(A color version of this figure is available in the online journal.)

of the disks using the IRAC colors from Megeath et al. (2004) and Allen et al. (2004).

For comparison, in panels (C) and (D) of Figure 4, we plot the IRAC detections with no X-ray counterpart: we note that sources with and without infrared excess in the CMD of panel (D) reach [3.6] $16 \mathrm{mag}$, while in the CMD of panel (B) sources without excess have $3.6 \mu \mathrm{m}$ magnitudes down to $15.5 \mathrm{mag}$, and only few sources with excess have $3.6 \mu \mathrm{m}$ magnitudes larger than 14 mag. The density distribution ${ }^{12}$ of the cluster members is shown in panels (A) and (B) of Figure 5. The distribution peaks within $100^{\prime \prime}$ from the center of the cluster. In panels (C) and (D) of Figure 5 the density distribution of infrared sources without an X-ray counterpart is shown. Out of 593 sources detected in ch1 and ch2 without an X-ray counterpart, we find 248 IRAC excess sources. ${ }^{13}$ The distribution of sources with excess is similar to the distribution of the cluster members, while the flat distribution of sources without excess suggests that most of them are not related to the cluster. For this reason, infrared sources without X-ray counterpart but with excess emission have been

\footnotetext{
${ }^{12}$ Defined as in Section 3.1.

13 Defined as [3.6]-[4.5] $\geqslant 0.2$.
}

classified as cluster member candidates. ${ }^{14}$ Tables 4 and 5 give positions and IRAC fluxes of the cluster member candidates (i.e., infrared sources with excess without X-ray counterpart).

\section{AGE AND MASS DISTRIBUTION OF THE CLUSTER MEMBERS}

In this section we estimate the age, the mass distribution, and the completeness of the cluster members of IC 1795, defined in Section 4 as infrared sources with an X-ray counterpart.

\subsection{Age of the Cluster}

The ages of PMS associations are usually estimated by comparing the location of the association members in a CMD or Hertzsprung-Russell diagram, to isochrones resulting from the predictions of PMS evolutionary model grids. This comparison usually makes use of dereddened optical or near-infrared photometry unaffected by the presence of a protoplanetary disk, but optical spectroscopy of most of the cluster members, to quantify the differential reddening to each source, is not available.

\footnotetext{
${ }^{14}$ See Section 3.3 for a possible extragalactic contamination.
} 

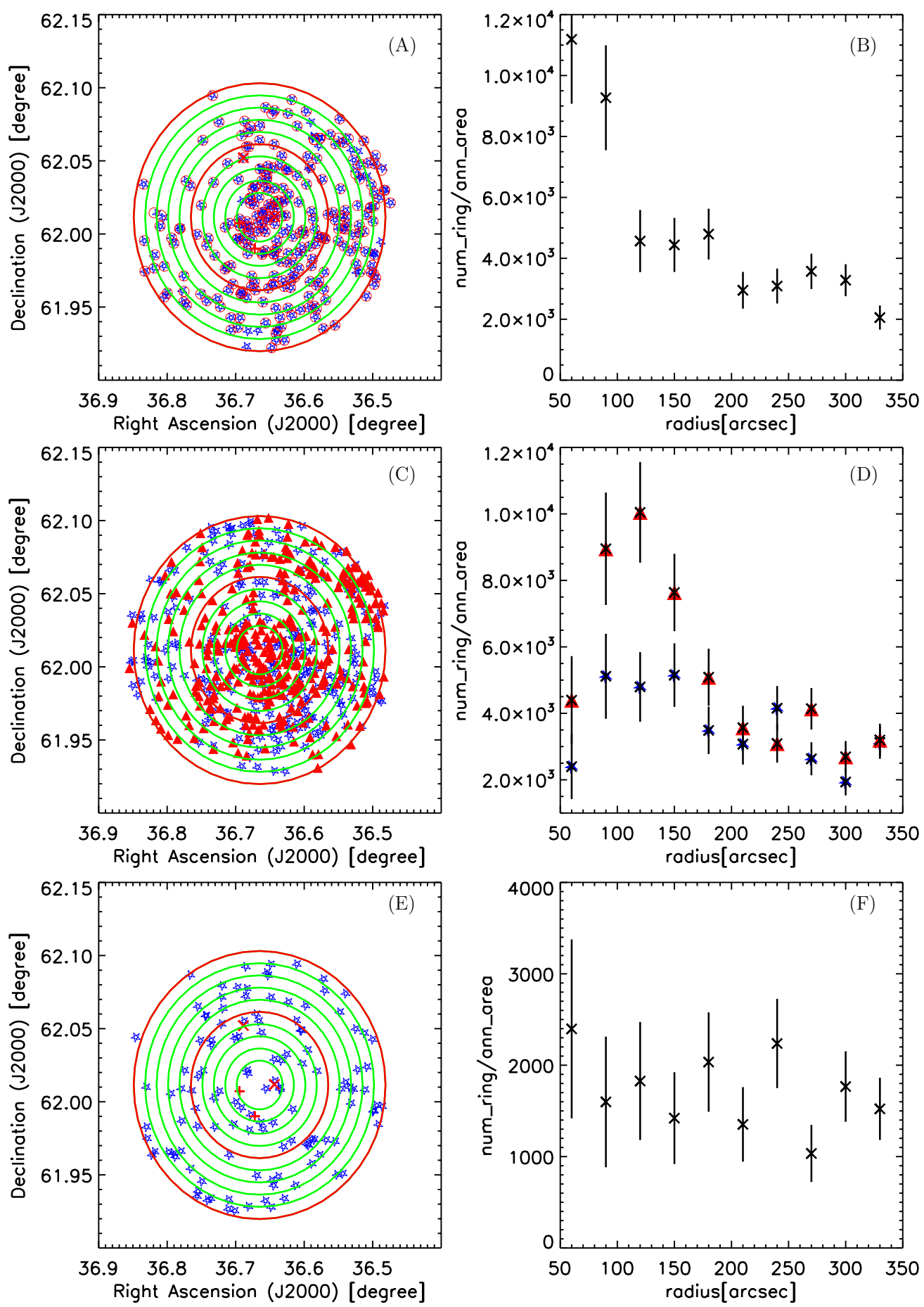

Figure 5. Spatial density distribution of all the IR sources (left column: A, C, E), and their spatial density distribution as a function of the distance from the center of the cluster (right column: B, D, F). The green circles represent annuli from $60^{\prime \prime}$ from the center of the cluster to $330^{\prime \prime}$ with a spacing of $30^{\prime \prime}$. The " $x$ " and " + " symbols represent the positions of the $\mathrm{O}$ and B stars in the cluster. (A and B) Infrared sources with an X-ray counterpart. (C and D) Infrared sources without an X-ray counterpart: the triangles represent sources with excess and the stars sources without excess. (E and F) X-ray sources without infrared counterpart.

(A color version of this figure is available in the online journal.)

The positions of the sources in these CMDs can thus be either affected by differential extinction or by a spread in age, as well as by undetected binarity.

However, for IC 1795 members we do have extensive 2MASS $J$ band and IRAC infrared [3.6] and [4.5] photometry. Thus, to optimize the isochronal fitting of IC 1795 members, we first look for the best magnitude and color to distinguish between sources with and without infrared excess. This is needed to clean the CMD of the infrared-excess sources.
The $J$ magnitude is not affected by infrared excess (which in the different classes of disks would usually start, at least, from the $H$ band) and is sensitive to the stellar mass. The first CMD analyzed used the $J$ versus $J-H$ magnitudes (Figure 6(A)). However, the disadvantage is that the $J$ and $H$ magnitudes are strongly affected by extinction along the line of sight. This represents a serious problem in particular working on a cluster at more than $1 \mathrm{kpc}$ in distance such as IC 1795 . Overlaid on the CMD in Figure 6(A) are the $3 \mathrm{Myr}$ and $5 \mathrm{Myr}$ 
Table 4

Cluster Member Candidates: Designation and Positions of IRAC Sources with Excess without X-ray Counterpart

\begin{tabular}{ccccc}
\hline \hline $\begin{array}{c}\text { ID } \\
\text { IRAC- }\end{array}$ & $\begin{array}{c}\text { R.A. } \\
(\mathrm{deg})\end{array}$ & $\begin{array}{c}\text { R.A. Error } \\
10^{-4}(\mathrm{deg})\end{array}$ & $\begin{array}{c}\text { Decl. } \\
(\mathrm{deg})\end{array}$ & $\begin{array}{c}\text { Decl. Error } \\
10^{-4}(\mathrm{deg})\end{array}$ \\
\hline $02270033+6205291$ & 36.751377 & 1.09 & 62.091415 & 1.12 \\
$02271605+6200506$ & 36.816875 & 0.43 & 62.014042 & 0.44 \\
$02271147+6201578$ & 36.797775 & 0.57 & 62.032722 & 0.60 \\
$02271512+6200152$ & 36.813011 & 0.45 & 62.004215 & 0.46 \\
$02271175+6158265$ & 36.798965 & 0.65 & 61.974022 & 0.67 \\
$02270991+6158521$ & 36.791275 & 1.63 & 61.981152 & 1.73 \\
$02264351+6205302$ & 36.681278 & 3.98 & 62.091713 & 3.92 \\
$02264566+6204469$ & 36.690266 & 0.60 & 62.079685 & 0.60 \\
$02270243+6200092$ & 36.760136 & 1.02 & 62.002544 & 1.05 \\
$02263985+6205356$ & 36.666031 & 5.19 & 62.093216 & 5.15 \\
\hline
\end{tabular}

(This table is available in its entirety in a machine-readable form in the online journal. A portion is shown here for guidance regarding its form and content.)

isochrones obtained using the FRANEC evolutionary code (see Appendix A). The isochrones are reddened with $A_{V}=2 \mathrm{mag}$ which is the minimum extinction expected for a cluster at a distance of $2 \mathrm{kpc}$. Without any information on a possible differential extinction within the cluster, from this CMD it is not possible to distinguish between the different isochrones. The positions of the sources can be either affected by additional extinction or by a spread in age, as well as by undetected binarity. Different CMDs, combining in different ways the $J, 3.6 \mu \mathrm{m}$, and $4.5 \mu \mathrm{m}$ magnitudes, have been further compiled. The advantage of using the $J-[3.6]$ or $J-[4.5]$ combinations is that photospheric and infrared excess sources are clearly separated in color. The advantage of using the [3.6]-[4.5] combination instead is that at these wavelengths the effect of interstellar extinction is lower (see the module of the extinction $A_{V}$ in Figure 6). We thus use the [3.6] versus [3.6]-[4.5] CMD to distinguish between objects with and without infrared excess, taking advantage of the negligible extinction at these wavelengths.

In Figure 6 (panel (B)), we show the [3.6]-[3.6]-[4.5] CMD of the Class III cluster members, together with the $1 \mathrm{Myr}, 3 \mathrm{Myr}$, and $5 \mathrm{Myr}$ isochrones. In this CMD, the only parameters that determine the vertical position of the Main-Sequence Turn-On (MSTON; see Appendix A) are the age and the distance of the
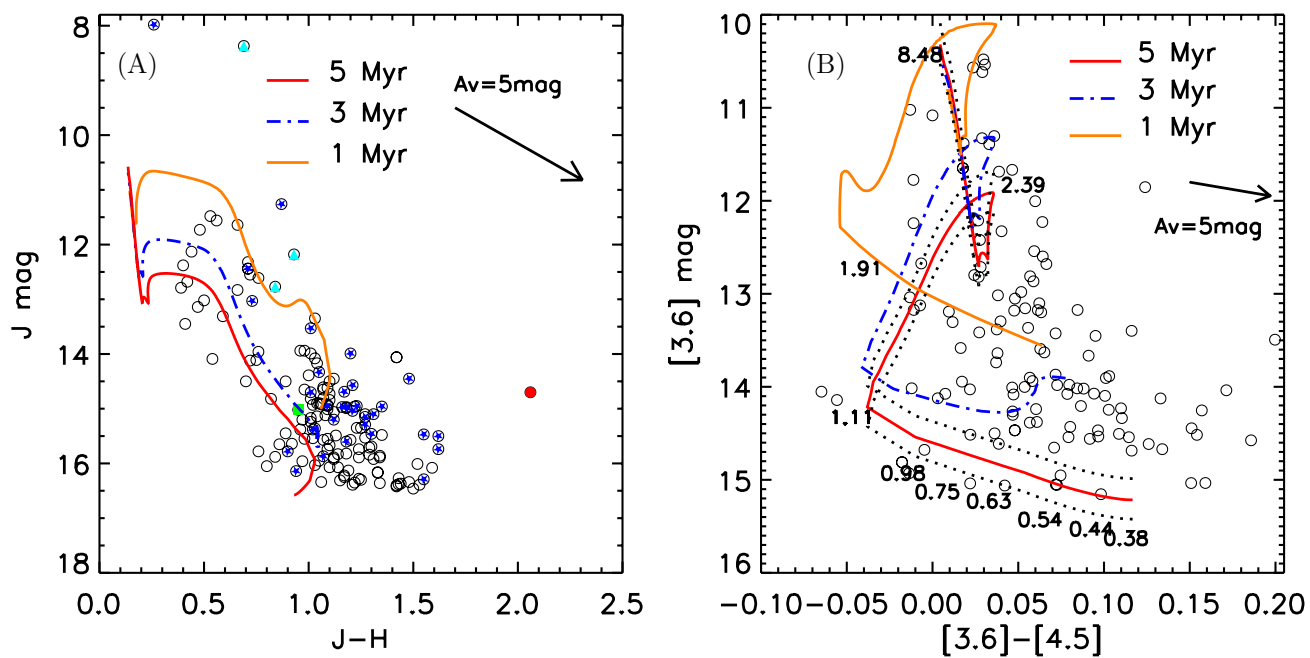

Figure 6. (A) CMD of the cluster members using the 2MASS magnitudes. Overlaid are the $1 \mathrm{Myr}, 3 \mathrm{Myr}$, and $5 \mathrm{Myr}$ isochrones (shifted at a distance of the cluster of $2 \mathrm{kpc}$ and $A_{v}=2 \mathrm{mag}$ ). The different symbols and colors are as in Figure 4. (B) Close up of CMD in panel (B) in Figure 4 around sources without infrared excess (empty circles) in the IRAC CMD (panel (B) in Figure 4). Overlaid are the $1 \mathrm{Myr}, 3 \mathrm{Myr}$, and $5 \mathrm{Myr}$ isochrones (shifted at a distance of the cluster of $2 \mathrm{kpc}$ and $A_{v}=$ $2 \mathrm{mag}$ ). The dotted lines represent the $5 \mathrm{Myr}$ isochrones but shifted at a maximum and minimum distance of 2.2 and $1.8 \mathrm{kpc}$. The numbers along the $5 \mathrm{Myr}$ isochrone represent the corresponding masses (in $M_{\odot}$ ). The arrow in panels (A) and (B) represents an extinction $A_{v}=5$ mag.

(A color version of this figure is available in the online journal.)

Table 5

Cluster Member Candidates: IRAC Sources with Excess without X-ray Counterpart

\begin{tabular}{cccccccrrr}
\hline \hline $\begin{array}{c}\text { ID } \\
\text { IRAC- }\end{array}$ & $\begin{array}{c}\text { FV } \\
(\mathrm{mJy})\end{array}$ & $\begin{array}{c}\text { FI } \\
(\mathrm{mJy})\end{array}$ & $\begin{array}{c}\text { FJ } \\
(\mathrm{mJy})\end{array}$ & $\begin{array}{c}\text { FH } \\
(\mathrm{mJy})\end{array}$ & $\begin{array}{c}\text { FK } \\
(\mathrm{mJy})\end{array}$ & $\begin{array}{c}\text { F3.6 } \\
(\mathrm{mJy})\end{array}$ & \multicolumn{1}{c}{$\begin{array}{c}\text { F4.5 } \\
(\mathrm{mJy})\end{array}$} & $\begin{array}{r}\text { F5.8 } \\
(\mathrm{mJy})\end{array}$ & \multicolumn{1}{c}{$\begin{array}{r}\text { F8.0 } \\
(\mathrm{mJJ})\end{array}$} \\
\hline $02270033+6205291$ & $\ldots$ & $\ldots$ & $\ldots$ & $\ldots$ & $\ldots$ & $1.1322 \pm 0.0061$ & $2.5148 \pm 0.0115$ & $3.2495 \pm 0.0278$ \\
$02271605+6200506$ & 1.0782 & 14.5794 & 208.2036 & 371.7919 & 619.3419 & $114.7894 \pm 0.3332$ & $120.9486 \pm 0.3271$ & $115.4599 \pm 0.3373$ & $73.2051 \pm 0.3046$ \\
$02271147+6201578$ & 0.0135 & 0.1829 & 0.9088 & 1.6684 & 2.2487 & $3.1107 \pm 0.0106$ & $3.0494 \pm 0.0110$ & $2.9472 \pm 0.0231$ & $\ldots$ \\
$02271512+6200152$ & 4.7239 & 24.0846 & 65.8398 & 68.9128 & 60.2463 & $32.1743 \pm 0.0982$ & $25.1779 \pm 0.0725$ & $17.0157 \pm 0.0942$ & $13.6489 \pm 0.1281$ \\
$02271175+6158265$ & $\ldots$ & $\ldots$ & $\ldots$ & $\ldots$ & $\ldots$ & $5.8413 \pm 0.0213$ & $4.9457 \pm 0.0146$ & $3.6184 \pm 0.0390$ & $\ldots$ \\
$02270991+6158521$ & $\ldots$ & $\ldots$ & $\ldots$ & $\ldots$ & $\ldots$ & $0.9234 \pm 0.0072$ & $0.7527 \pm 0.0044$ & $\ldots$ & $\ldots$ \\
$02264351+6205302$ & $\ldots$ & $\ldots$ & $\ldots$ & $\ldots$ & $\ldots$ & $0.2417 \pm 0.0040$ & $0.6026 \pm 0.0054$ & $\ldots$ & $\ldots$ \\
$02264566+6204469$ & $\ldots$ & $\ldots$ & $\ldots$ & $\ldots$ & $\ldots$ & $2.9971 \pm 0.0102$ & $3.9269 \pm 0.0142$ & $5.7818 \pm 0.0314$ & $9.6677 \pm 0.0823$ \\
$02270243+6200092$ & $\ldots$ & $\ldots$ & $\ldots$ & $\ldots$ & $\ldots$ & $1.4936 \pm 0.0078$ & $1.6670 \pm 0.0078$ & $\ldots$ & $\ldots$ \\
$02263985+6205356$ & $\ldots$ & $\ldots$ & $\ldots$ & $\ldots$ & $\ldots$ & $0.2056 \pm 0.0044$ & $0.1769 \pm 0.0040$ & $1.1781 \pm 0.0321$ & $4.1815 \pm 0.0902$
\end{tabular}

Notes. The IRAC flux errors refer to the covariance matrix calculated from the "best fit" of the PRF to the data and do not include calibation uncertainty ( $\sim 10 \%$ of the flux).

(This table is available in its entirety in a machine-readable form in the online journal. A portion is shown here for guidance regarding its form and content.) 

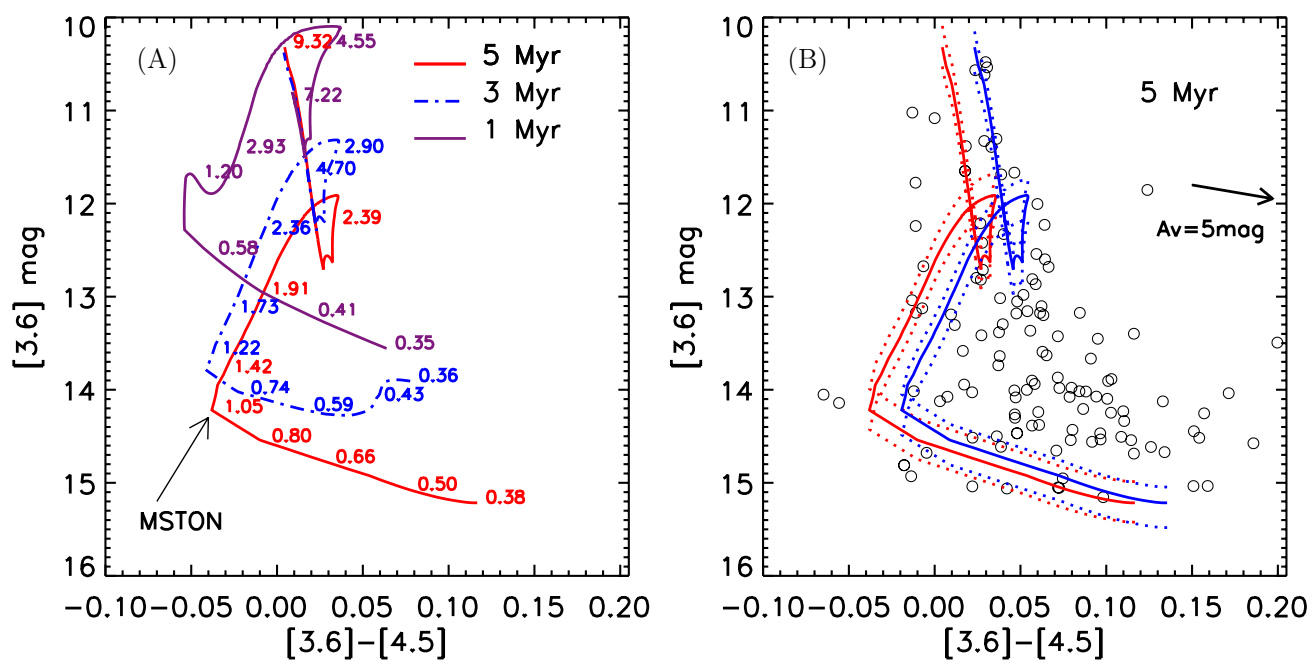

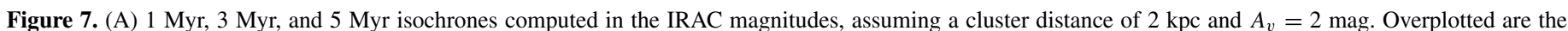

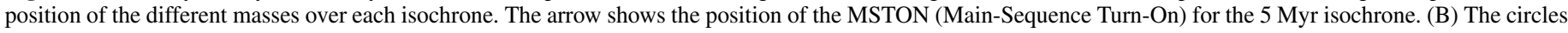

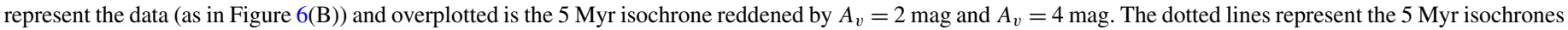
but shifted at a maximum and minimum distance of 2.2 and $1.8 \mathrm{kpc}$ (as in Figure 6(B)).

(A color version of this figure is available in the online journal.)

cluster. We note that at these wavelengths an error of $10 \%$ in distance correspond to an uncertainty of less than $1 \mathrm{Myr}$ in age and the reddening is unimportant because the reddening vector (see Figure 6, panel (B)) runs parallel to the low-mass population. We also investigate whether an additional extinction of $2 \mathrm{mag}$ in $V$ would change the position of MSTON and hence the conclusions about the upper limit on the age of the system. In Figure 7(B) the $5 \mathrm{Myr}$ isochrone is reddened by $A_{v}$ $=2 \mathrm{mag}$ and $A_{v}=4 \mathrm{mag}$. Taking into account the errors on the distance of the cluster (shown also in Figure 7(B)) and on the photometry of the single sources (the maximum error was 0.07 mag; see Section 3.2), we conclude that, within the errors, all the stars still lie close or above the 5 Myr isochrone. For these reasons the apparent age of the cluster can be determined using reddened photometry. From the MSTON we can conclude that the cluster is younger than $5 \mathrm{Myr}$ and it likely has an age between 3 and 5 Myr. This is consistent with an earlier estimate (Oey et al. 2005) which was obtained from the position in the H-R diagram of the early type sources of IC 1795 .

Due to the presence of other stars with a $3.6 \mu \mathrm{m}$ magnitude in the range of 12.5-14 mag which do not follow the shape of the isochrones, we investigate the possibility of a spread in age of the cluster members. We first note that the $1 \mathrm{Myr}$ isochrone does not reproduce the distribution of the observed sources on the CMD (Figure 6). If we presume that the cluster has a spread in age between 1 and $5 \mathrm{Myr}$ due to different episodes of star formation, sources with a $3.6 \mu \mathrm{m}$ magnitude in the range of $12.5-14 \mathrm{mag}$ would represent the younger population of the cluster, since for the $1 \mathrm{Myr}$ isochrone all stellar masses reside within this magnitude range (Figure 6). In this case, they would also have a different spatial distribution with respect to the older population with $3.6 \mu \mathrm{m}>14 \mathrm{mag}$. However, the random spatial distribution of the photospheric sources with different [3.6] mag argues against a significant spread in age in the cluster population caused by triggered episodes of star formation. A second argument against a young population present in the cluster is regarding the CMD using the 2MASS magnitudes (Figure 6, panel (A)). The overlaid isochrones are shifted assuming a distance to the cluster of $2 \mathrm{kpc}$ and $A_{v}=2 \mathrm{mag}$, which is the minimum extinction for a cluster at that distance. In this CMD the bulk of the sources are bluer and fainter than the $1 \mathrm{Myr}$ isochrone.

These arguments, however, do not exclude that a younger population might be present in the cluster and is viewed in projection over the older one. A possibility is that stars with $[3.6]=12-14$ are binaries. In Section 5.2, we test this hypothesis by including the binarity in a simulation of the theoretical luminosity function of the cluster.

\subsection{Luminosity and Mass Functions of IC 1795}

The comparison of the observed and theoretical luminosity function enables us to estimate the completeness limit of our Spitzer-Chandra survey.

The observed luminosity function is compiled using the $3.6 \mu \mathrm{m}$ magnitude as a tracer of the stellar photosphere. To have a description of the entire stellar population of IC 1795, the observed luminosity function includes cluster members with and without infrared excess and cluster member candidates (defined in Section 4.3). This approach is necessary because the disk fraction may be mass dependent (Section 6). We used the method described in Appendix B to determine the "photospheric" $3.6 \mu \mathrm{m}$ magnitude of the sources with infrared excess. The same has been done for the cluster member candidates.

We computed the theoretical luminosity function using a galactic initial mass function (IMF; Kroupa 2002) and the $4 \mathrm{Myr}$ and $5 \mathrm{Myr}$ isochrones, respectively. The simulated population is normalized to the observed stellar sample down to a certain completeness magnitude limit.

We compare the simulations obtained using a completeness value of $13.5 \mathrm{mag}, 13.75 \mathrm{mag}, 14 \mathrm{mag}, 14.25 \mathrm{mag}, 14.5 \mathrm{mag}$, and $15 \mathrm{mag}$ at $3.6 \mu \mathrm{m}$. We conclude that the completeness of our survey at $3.6 \mu \mathrm{m}$ is $14 \mathrm{mag}$.

We note, however, that for both ages of 4 Myr and $5 \mathrm{Myr}$, the simulations do not well reproduce the number of sources in the $3.6 \mu \mathrm{m}$ magnitude range of 13-14. This might be an effect of a binary population unresolved in the cluster. To test this, we ran simulations with $30 \%$ and $50 \%$ binary fractions, with primary and secondary masses drawn independently from the same IMF. The outcomes are only slightly different from our 

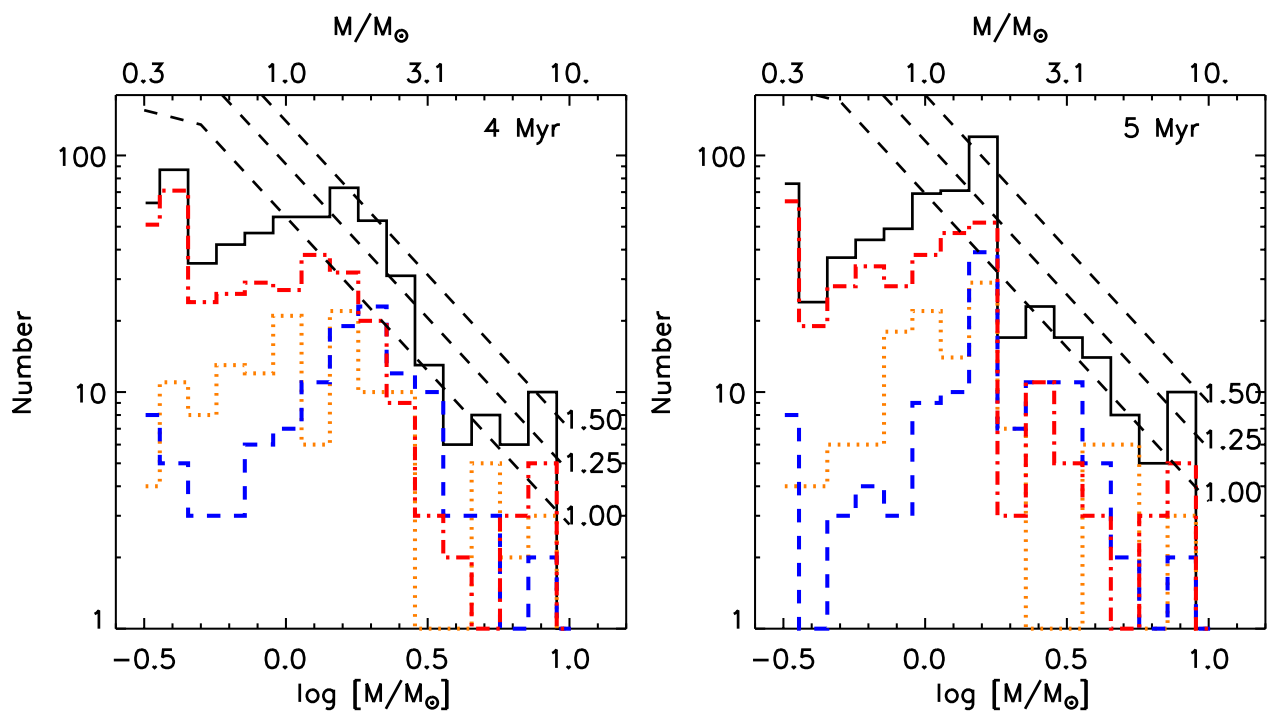

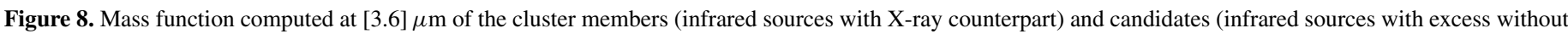

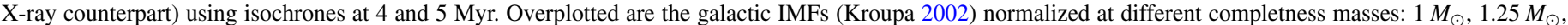

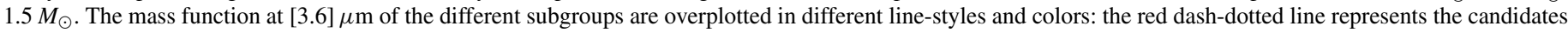

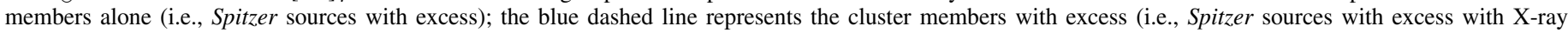
counterpart); the orange dotted line represents the cluster members without excess.

(A color version of this figure is available in the online journal.)

original calculations. This implies that the luminosity function is only marginally affected by binarity.

In order to convert the completeness expressed in magnitudes into the corresponding stellar mass value, the mass function of IC 1795 is now extracted.

Using the $4 \mathrm{Myr}$ and $5 \mathrm{Myr}$ isochrones, from the observed luminosity function we derived the stellar masses and hence construct the mass function for the cluster. We used the method described in Appendix $C$ to assign a stellar mass to each source. The resulting observed total mass function in shown in Figure 8 together with the mass functions of the single sub-groups considered for the total mass function: the cluster members ${ }^{15}$ without and with infrared excess and the cluster candidates. ${ }^{16}$ The theoretical IMF (Kroupa 2002) is normalized to the total number of stars down to $14 \mathrm{mag}$. We note that all the distributions peak at the same mass. This ensures that both the Spitzer and Chandra surveys have the same completeness. There is a large population of low-mass cluster candidates down to $0.4 M_{\odot}$ which lack an X-ray counterpart. The observed mass function falls below the theoretical IMF below $\sim 1.0 M_{\odot}$. This mass corresponds to the estimated completeness at $3.6 \mu \mathrm{m}$ of $14.0 \mathrm{mag}$.

Extrapolating the total population of IC 1795 down to a mass of $0.08 M_{\odot}$, the total cluster membership numbers $\sim 2000$ stars. This corresponds to a stellar density of $\sim 15 \mathrm{stars}^{-3} \mathrm{c}^{-3}$, which is several thousands lower than the density in the Trapezium cluster.

In summary,

1. IC 1795 has an age between 3 and 5 Myr consistent with the previous estimate of Oey et al. (2005),

2. the mass function is well reproduced by a standard IMF to a mass limit of $1 M_{\odot}-1.2 M_{\odot}$, and

3. the cluster population is estimated to be 2000 sources at a mass limit of $0.08 M_{\odot}$.

15 Spitzer sources with X-ray counterpart.

16 Spitzer sources with excess without X-ray counterpart.

\section{DISK EVOLUTION: EFFECT OF THE STELLAR MASS AND THE ENVIRONMENT}

In this section, the analysis of the disk fraction as a function of the stellar mass and distance from the center of the cluster is presented for the cluster members ${ }^{17}$ of IC 1795.

\subsection{Disk Fraction versus Stellar Mass and Spatial Distribution}

We examine the mass dependence of disk emission of the 3-5 Myr old cluster IC 1795. Since only few stars have been spectrally classified so far (Oey et al. 2005), and a detailed spectral classification of the cluster members is in preparation ( $\mathrm{J}$. S. Kim et al. 2011, in preparation), the disk fraction is computed using the $3.6 \mu \mathrm{m}$ and the $J$ magnitudes as a tracer of stellar photosphere (Figures 9 and 10; for details see Section 5.2).

In order to calculate the fraction of sources with and without infrared excess, we follow the method described in Appendix B. We note that, between [3.6] $=10-12$, the disk fraction rapidly increases from $20 \%$ to $50 \%$, and then it remains constant at $60 \%-40 \%$ between 12 and $14.5 \mathrm{mag}$. Constructing a $2 \times 2$ contingency table of IR excess for the [3.6] = 10-12 and 12-14 mag ranges, we formulate a hypothesis test with the null hypothesis that IR excess does not depend on [3.6] or, equivalently, on stellar mass. A Fisher's exact test applied to the contingency table indicates that the probability of this null hypothesis is $P=0.005$. The test is implemented by the $R$ statistical software package (R Development Core Team 2009). We conclude that the effect is statistically reliable.

Since $\sim 80 \%$ of our IRAC sources have a near infrared counterpart, we used the $J$ magnitude as a proxy for stellar mass (see, e.g., Hernández et al. 2007). These bands have the advantage of being relatively unaffected by disk excess emission. However, they are strongly affected by extinction. The 4 Myr isochrone (see Section 5.1) has been used to infer masses from the $J$ magnitudes. In this band the relation between mass and magnitude is almost unique (see Figure 11).

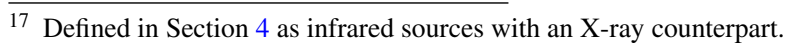



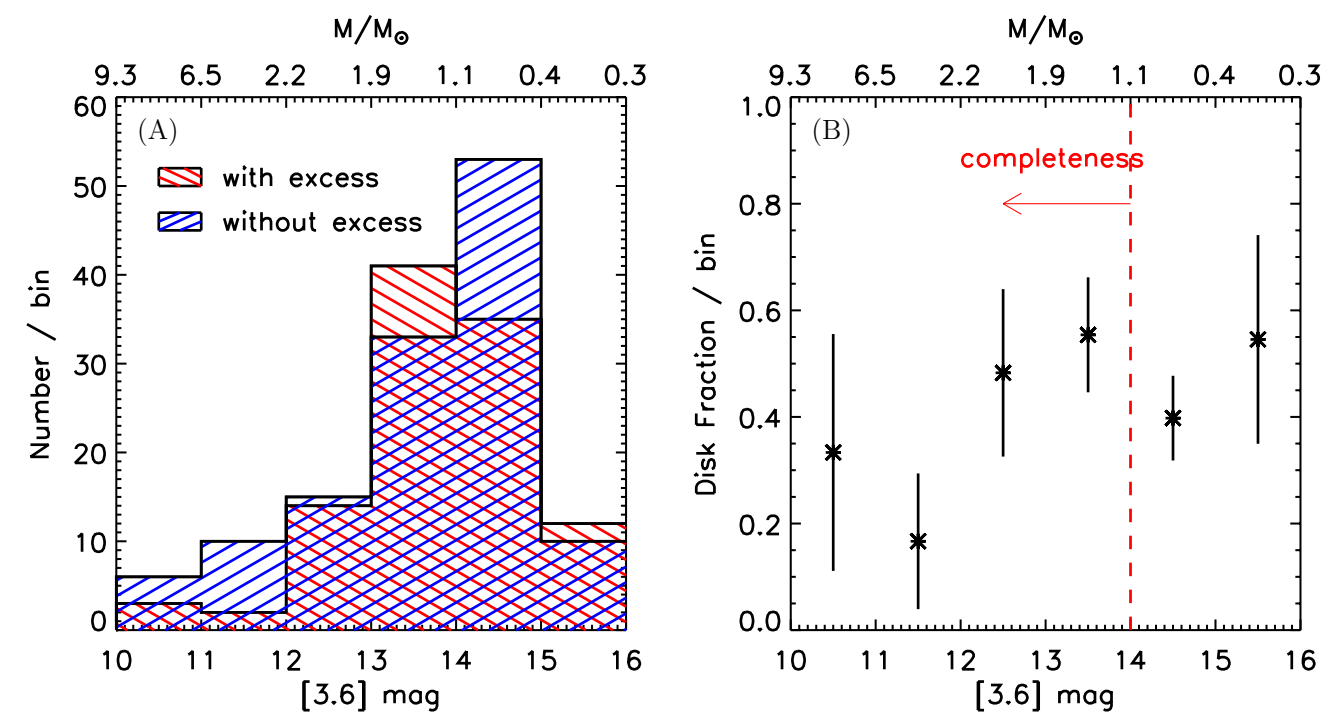

Figure 9. (A) Histogram of cluster member (e.g., IRAC sources with an X ray counterpart) sources with excess (i.e., with [3.6]-[4.5] > 0.2) and without excess with a bin of 1 mag. (B) Disk fraction computed as ratio of the sources with excess per [3.6] bin and the total number of sources per bin. The corresponding masses are computed using the $4 \mathrm{Myr}$ isochrone.

(A color version of this figure is available in the online journal.)

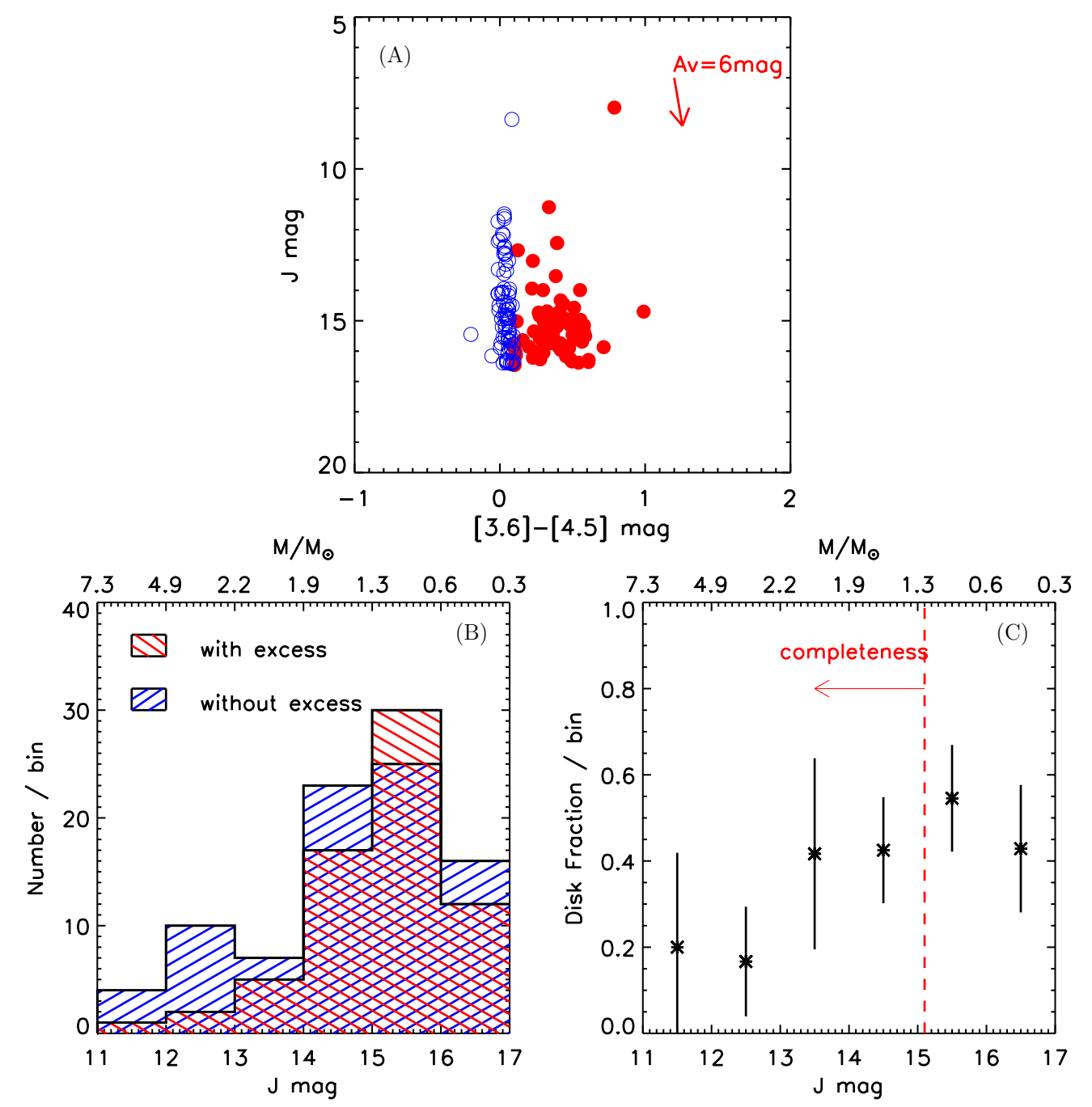

Figure 10. (A) $J-[3.6]-[4.5]$ CMD. The arrow represents an extinction $A_{V}=6$ mag. Filled circles represent sources with excess (i.e., with [3.6]-[4.5] $>0.2$ ), and empty circles represent sources without excess. (B) Number of sources with and without excess per bin of $J=1$ mag. (C) Disk fraction computed as ratio of the sources with excess per $J$ magnitude bin and the total number of sources per bin. The corresponding masses are computed using the 4 Myr isochrone.

(A color version of this figure is available in the online journal.) 

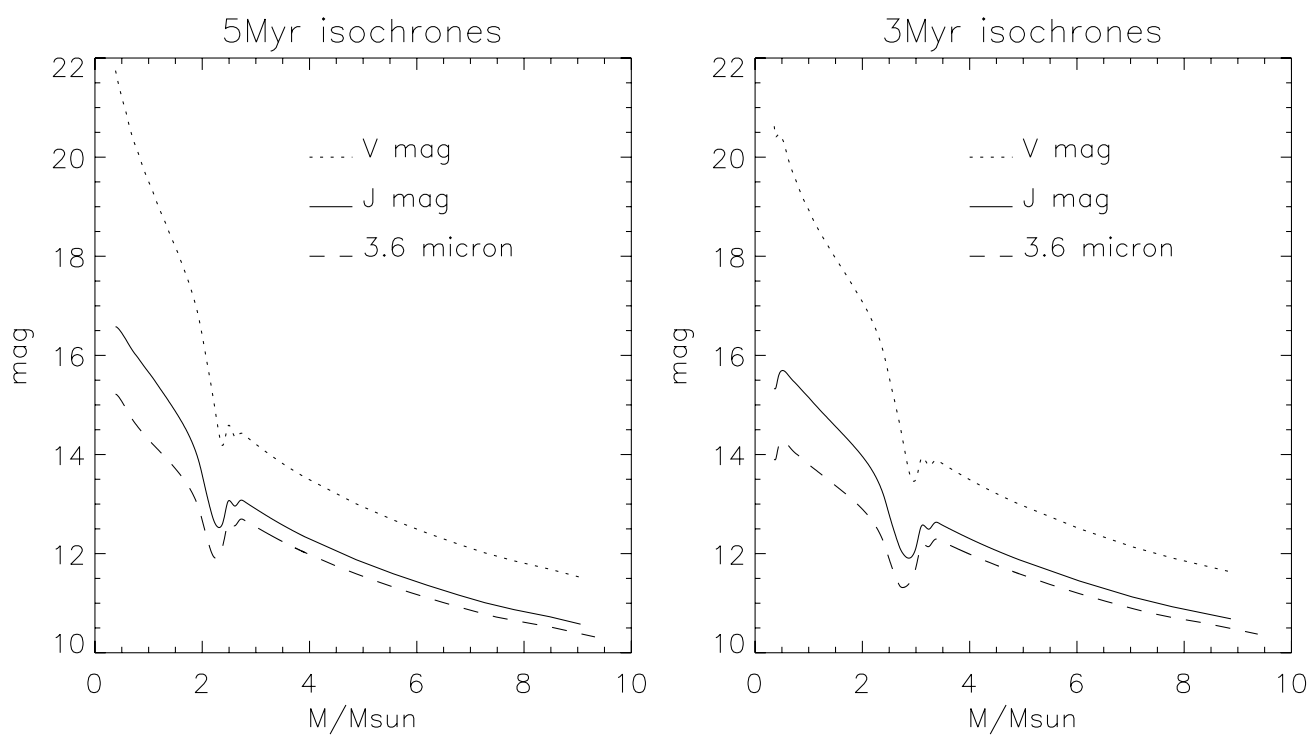

Figure 11. Relation between the $V, J$, and $3.6 \mu \mathrm{m}$ magnitudes and the stellar masses computed by the $5 \mathrm{Myr}$ (left) and $3 \mathrm{Myr}$ (right) isochrones.

In Figure 10 (panel (A)), we show the CMD using the $J$ magnitude as a photospheric tracer and the [3.6]-[4.5] IRAC colors to define the sources with and without infrared excess. After the histograms of sources with and without excess (panel (B) of Figure 10), the disk fraction is shown in panel (C). This is computed as the ratio of sources with and without excess in bins of 1 mag. The errors are computed as the square root of the number of sources in each bin. The corresponding mass is reported in the upper $x$-axis.

The disk fraction is found to increase from $20 \%$ to $60 \%$ for $J$ magnitudes from 11 to 15 . This corresponds approximately to masses between 8.4 and $0.8 M_{\odot}$. The disk fraction remains constant around 50\% toward lower masses. An increasing frequency of disks is obvious down to 15 mag even after taking into account the uncertainty in the disk fraction.

Taking into account solar-type stars only with masses $\sim 1 M_{\odot}$ (our completeness level, Section 5.2), the disk fraction is 50\%.

This value is consistent with the disk fraction of solar-type stars previously computed with the $3.6 \mu \mathrm{m}$ magnitude.

A dependency of the disk fraction on the stellar mass was found in Spitzer surveys of young clusters with low- and high-mass members (e.g., Hernández et al. 2007; Carpenter et al. 2006; Currie et al. 2007; Lada et al. 2006; Luhman et al. 2010). The general finding is that massive stars seem to lose their disk earlier than lower mass stars. This was also found in clusters older than 3 Myr by Kennedy \& Kenyon (2009), using published optical spectra and infrared excess data.

In young clusters the disk fraction may depend also on the spatial position. If triggered star formation took place in IC 1795 itself, a different spatial distribution of sources with and without disks and with an age gradient is expected (e.g., Cepheus B, Tr 37, Getman et al. 2009; Sicilia-Aguilar et al. 2005, 2006). In Figure 12, we show the spatial density distribution of sources with and without infrared excess.

The disk fraction $\left(f_{d}\right)$ is defined as the ratio between the number of sources with disks $\left(N_{\text {disk }}\right)$ and the total number of sources (with and without disks; $N_{\text {tot }}$ ). The fraction $f_{d}$ was computed in consecutive rings. Within the uncertainties, the disk fraction remains constant across the IC 1795 region at about $50 \%$.
This result argues against a triggered star formation scenario for IC 1795. A constant disk fraction as a function of the distance from the center of the cluster was also found, e.g., in $\sigma$ Ori (Oliveira et al. 2006) using $K$ - and $L$-band observations. This result is however in contrast with Hernández et al. (2007) who instead found an evidence for a higher disk fraction near the cluster center for $\sigma$ Ori.

\subsection{Influence of the O Star in the Cluster: Disk Photoevaporation and Cluster Dynamics}

In Figure 1, we note that the 06.5V and 09.7I stars lie at $39^{\prime \prime}$ west and $120^{\prime \prime}$ north, respectively, from the projected spatial center of the cluster defined in Section 3.1. In order to study the influence of the $\mathrm{O}$ stars in the cluster we have re-centered the cluster on each $\mathrm{O}$ star. In both cases, the spatial distribution of the disk fraction observed is almost constant with distances from each $\mathrm{O}$ star including within the first $60^{\prime \prime}$ (which corresponds to $0.58 \mathrm{pc}$ at the cluster distance).

This result is not in agreement with previous works of Balog et al. (2007) and Mercer et al. (2009). From the analysis of the 2-3 Myr NGC 2244 cluster Balog et al. (2007) found a smaller disk fraction within $0.5 \mathrm{pc}$ from the $\mathrm{O}$ stars, including the two highest-mass O stars, HD 46223 (O5) and HD 46150 (O6), of the cluster.

Johnstone et al. (1998) predicted an absence of disks in systems proximate to $\mathrm{O}$-stars, a prediction supported observationally by Hernández et al. (2008) who found no evidence for primordial disks within $0.75 \mathrm{pc}$ of a high-mass binary (consisting of an O 7.5 star and a Wolf-Rayet star) in the $\gamma$ Velorum star-forming region.

However, theoretical expectations predict the photoevaporation of the outer part of the disk ( $>5 \mathrm{AU}$ ), while the inner regions (traced by Spitzer/IRAC), where the escape velocity exceeds the sound speed of the ionized gas, cannot be evaporated. The dissipation of such a part of the disk proceeds via viscous transport of material from the inner to the outer disk (e.g., Adams et al. 2004). Models predict that a high-mass star introduces an external UV radiation which can photoevaporate disks only within 0.3-0.7 pc (e.g., Johnstone et al. 1998; Adams et al. 2004; Clarke 2007; Gorti et al. 2009). Finally, we 


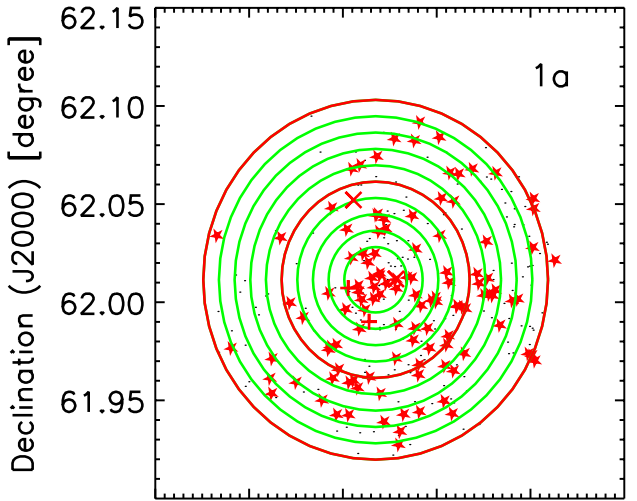

$\begin{array}{lllll}36.9 & 36.8 & 36.7 & 36.6 & 36.5\end{array}$

Right Ascension (J2000) [degree]

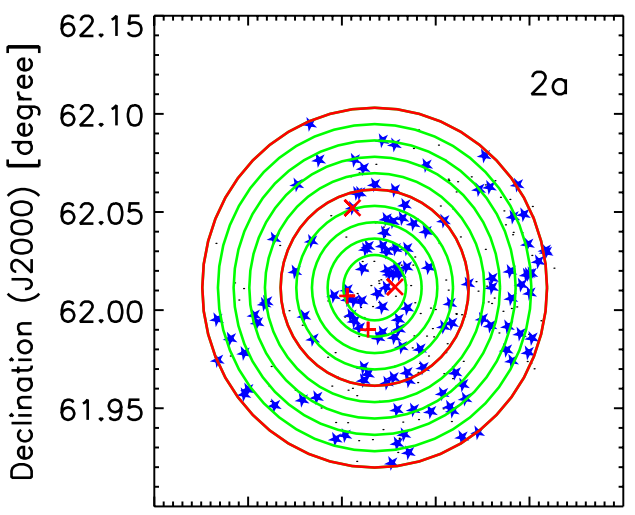

$\begin{array}{lllll}36.9 & 36.8 & 36.7 & 36.6 & 36.5\end{array}$ Right Ascension (J2000) [degree]

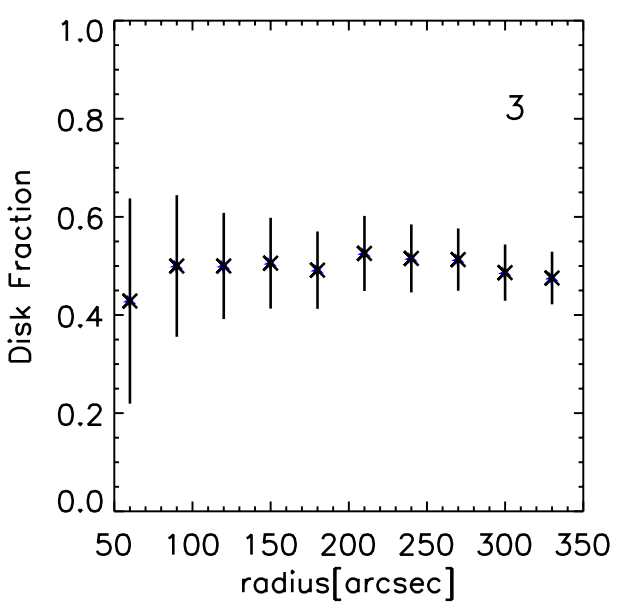

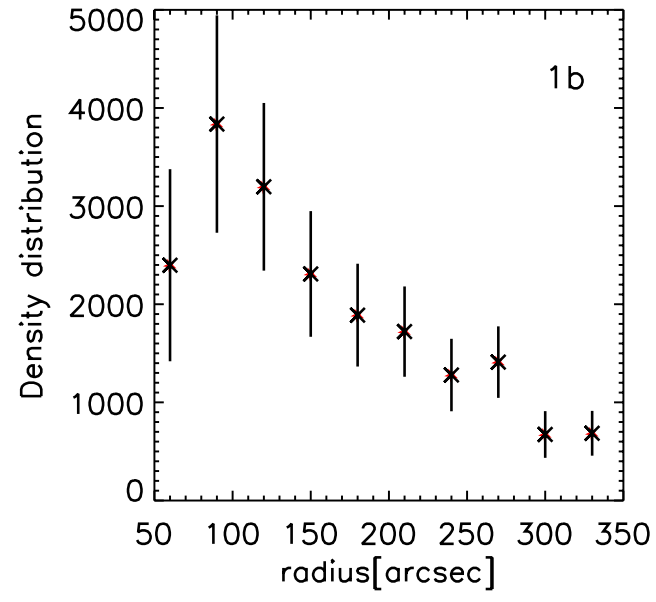

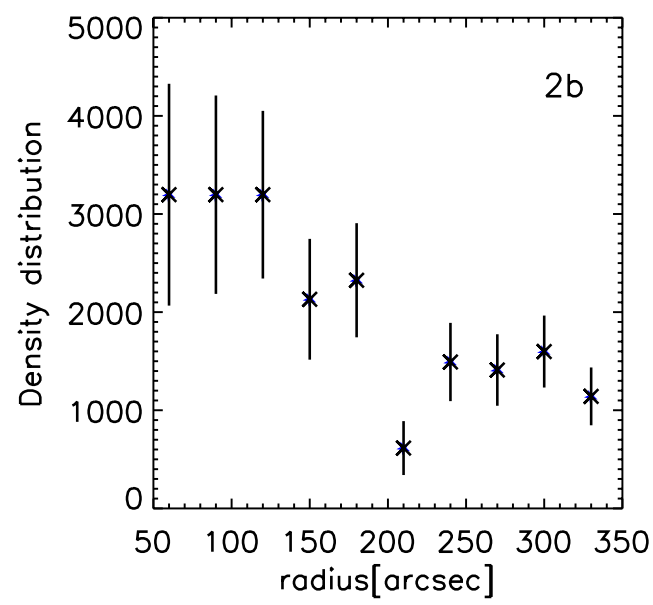

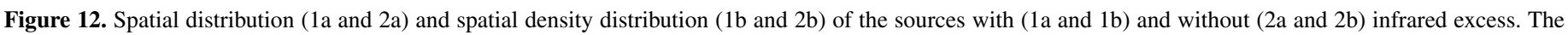

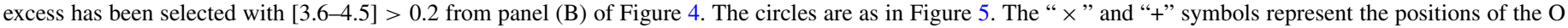
and B stars in the cluster. In panel 3 the disk fraction is computed as a function of the distance from the center of the cluster.

(A color version of this figure is available in the online journal.)

investigate whether mass segregation acted on the dynamics of the cluster.

In IC 1795 the high-mass stars are neither in the center of the cluster nor are they preferentially concentrated in a particular region compared to lower mass members (see Figure 1). The exception is represented by the $06.5 \mathrm{~V}$ star which is only $38^{\prime \prime}$ away from the cluster center. Since the spatial distribution of high-mass stars does not allow us to conclude if mass segregation has taken place in IC 1795, we check whether the cluster is in dynamical relaxation. The timescale of mass segregation can be approximated by the relaxation time $\left(t_{\text {relax }}{ }^{18}\right)$. Adopting a radius of the cluster $R \sim 3.2 \mathrm{pc}$ (see Section 3.1) and using a rough estimate for the unmeasured velocity dispersion $v_{\text {disp }} \sim 3 \mathrm{~km} \mathrm{~s}^{-1}$ (Binney \& Tremaine 1987), and $N \sim 2000$ stars (see Section 5.2), we obtain $t_{\text {relax }} \sim 68$ Myr for IC 1795.

\footnotetext{
$18 t_{\text {relax }} \approx(N / 8 \ln N) t_{\text {cross }}$, where $t_{\text {cross }}=2 R / v_{\text {disp }}$ is the crossing timescale and $v_{\text {disp }}$ is the velocity dispersion (Bonatto et al. 2006) in which a cluster reaches some level of kinetic energy equipartition, with the massive stars sinking to the core and low-mass stars moving to the cluster halo (e.g., Bonatto et al. 2006).
} 


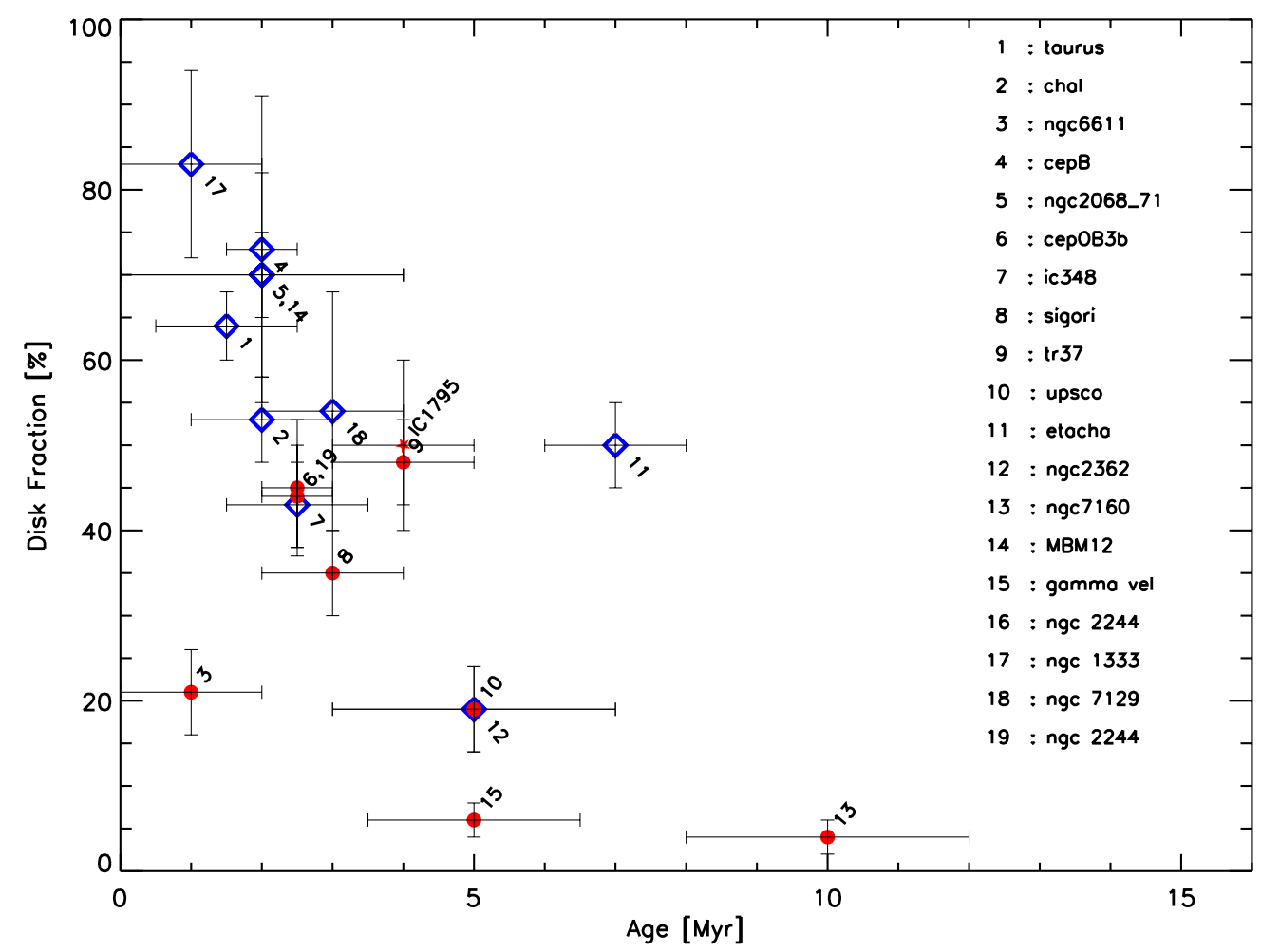

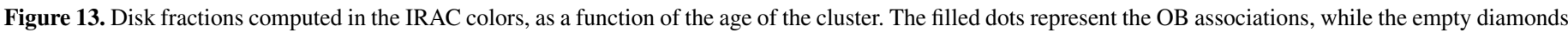
represent the low-mass star-forming regions.

(A color version of this figure is available in the online journal.)

As the cluster age is $\sim 17$ times less than its relaxation time, this calculation supports the observational evidence that mass segregation has not occurred in IC 1795. A similar result has been found in the 2-3 Myr old cluster NGC 2244 by Wang et al. (2008, 2009). However, in young rich clusters, massive stars are usually concentrated in the center (e.g., ONC; Hillenbrand et al. 1998).

\subsection{Disk Evolution in Low- and High-mass Environments}

In this section, the disk fraction of IC 1795 is compared with the results obtained in other low- and high-mass star-forming regions with the aim of investigating the influence of high-mass stars on the disk dissipation timescale. In particular, using the mid-infrared wavelength range, which traces the CS dust at a few AU from the star, it is possible to constrain the dissipation timescale of the region of the CS disks where planets form.

The disk fractions computed using the IRAC/Spitzer colors have been compiled from the literature and compared with the result obtained on IC 1795. The clusters considered are listed in Table 6. The total disk fraction from the individual studies is presented highlighting the completeness mass range of their work. When available, the disk fraction only in the intermediate-high-mass range is also reported. When the errors on the disk fractions $(\Delta f)$ are not available in the literature, we used $\Delta f=\frac{1}{\sqrt{N_{\text {TOT }}}}$, where $N_{\text {TOT }}$ is the total number of cluster members.

The general trend of disk dissipation in low-mass and highmass environments (see Figure 13) suggests that in young clusters (1 Myr) the fraction of disks is about $90 \%-80 \%$. By about 3-4 Myr the fraction of disks is reduced to 50\%-40\%. At 5 Myr the disk fraction drops to $20 \%$ while after 10 Myr almost all disks are dissipated.
Apart from the general trend previously described, the disk fraction of clusters containing more than $5 \mathrm{O} / \mathrm{B}$ stars shows mass dependent effects. For the $\mathrm{O}$ and $\mathrm{B}$ stars, the disk fraction decreases to $30 \%$ in the first $3 \mathrm{Myr}$, while in the low-mass environment it is still $60 \%-85 \%$.

Our result is consistent with the disk fraction found in cluster Tr 37 (Sicilia-Aguilar et al. 2006), an OB association with an age similar to that of IC 1795.

Most of the disk fractions from the literature are computed for clusters within $1 \mathrm{kpc}$. IC 1795 is at $\sim 2 \mathrm{kpc}$ and our study is complete to $\sim 1 M_{\odot}$, which corresponds to early-K spectral types. Therefore, a comparison between the disk fraction of $50 \% \pm 10 \%$ found for IC 1795 and that of other clusters might be misleading, e.g., for lower masses where our survey is incomplete, we expect to miss a significant fraction of the photospheric population.

A number of bias effects in compiling disk fractions from the literature (e.g., the completeness of the cluster memberships, the uncertainty on the age, and the disk fractions not specified in different mass range) have been carefully highlighted, since they can affect our conclusions. Given all uncertainties, we find that IC 1795 follows the smooth decline of the disk fraction with age observed for other clusters.

\section{SUMMARY}

In this paper, we presented a deep Spitzer/IRAC survey of the OB association IC 1795.

Combining the infrared Spitzer/IRAC observations with a deep X-ray Chandra/ACIS survey we carefully establish the cluster membership of the cluster. Compared to previous optical based studies on young clusters, this is the only method which allows for a determination of the disk fraction for cluster 
Table 6

Disk Fraction of Solar Type Stars

\begin{tabular}{|c|c|c|c|c|c|c|}
\hline Cluster & $\begin{array}{l}\text { Age } \\
(\mathrm{Myr})\end{array}$ & $\begin{array}{c}\text { Distance } \\
\text { (pc) }\end{array}$ & $\begin{array}{c}\text { Disk fraction } \\
\text { SpT }<\text { K6 }\end{array}$ & $\begin{array}{c}\text { Disk Fraction } \\
\text { Total }\end{array}$ & $\begin{array}{l}\text { Notes on the } \\
\text { Completeness }\end{array}$ & Ref. Disk Fraction \\
\hline NGC 1333 & $1 \pm 1$ & 280 & $\ldots$ & $83 \% \pm 11 \%(87 / 72)$ & $>0.08 M_{\odot}$ & Gutermuth et al. (2008) \\
\hline Taurus & $1.5 \pm 1.5$ & 140 & $80 \% \pm 15 \%(33 / 41)$ & $64 \% \pm 5 \%(226 / 352)$ & K0-M8 & Luhman et al. (2010) \\
\hline Cep B(embedded) & $1.5 \pm 0.5$ & 725 & $80 \% \pm 25 \%(12 / 15)$ & $73 \% \pm 18 \%(22 / 30)$ & $>0.5 M_{\odot}$ & Getman et al. (2009) \\
\hline NGC $6611^{\mathrm{b}}$ & $1 \pm 1$ & 1750 & $\ldots$ & $21 \% \pm 5 \%^{\mathrm{c}}$ & $0.3-3 M_{\odot}$ & Guarcello et al. $(2007,2009)$ \\
\hline Cha I & $2 \pm 1$ & 165 & $74 \% \pm 12 \%(14 / 19)$ & $53 \% \pm 7 \%(107 / 202)$ & K0-M8 & Luhman et al. (2008) \\
\hline NGC 2068/71 & $2 \pm 1.5$ & 400 & $\ldots$ & $79 \% \pm 12 \%(54 / 69)$ & G6-M6 & Flaherty \& Muzerolle (2008) \\
\hline NGC 7129 & $2 \pm 1$ & 1000 & $\cdots$ & $54 \% \pm 14 \%(27 / 50)$ & $>0.08 M_{\odot}$ & Gutermuth et al. (2004) \\
\hline MBM 12 & $2 \pm 2$ & 275 & $\ldots$ & $70 \% \pm 12 \%(7 / 12)$ & K3-M5 & Meeus et al. (2009) \\
\hline IC 348 & $2.5 \pm 1$ & 320 & $20 \% \pm 8 \%(7 / 35)$ & $43 \% \pm 6 \%(128 / 294)$ & K0-M8 & Luhman et al. (2010) \\
\hline Cep OB $3 b^{a}$ & $2.5 \pm 0.5$ & 725 & $42 \% \pm 14 \%(21 / 50)$ & $45 \% \pm 8 \%(63 / 140)$ & $>0.5 M_{\odot}$ & Getman et al. (2009) \\
\hline NGC $2244^{\mathrm{b}}$ & $2.5 \pm 0.5$ & 1500 & $\ldots$ & $44 \% \pm 6 \%(164 / 374)$ & Not defined & Balog et al. (2007) \\
\hline$\sigma$ Ori $^{\mathrm{a}}$ & $3 \pm 1$ & 350 & $\ldots$ & $35 \% \pm 5 \%(117 / 336)$ & K4-M5 & Hernández et al. (2007) \\
\hline NGC 7129 & $3 \pm 1$ & 1000 & $\ldots$ & $54 \% \pm 14 \%(27 / 50)$ & $>0.2 M_{\odot}$ & Gutermuth et al. (2004) \\
\hline $\operatorname{Tr} 37^{\mathrm{a}}$ & $4 \pm 1$ & 900 & $67 \% \pm \%(33 / 49)$ & $48 \%(62 / 129)$ & K0-M2 & Sicilia-Aguilar et al. (2006) \\
\hline Upper $\mathrm{Sco}^{\mathrm{a}}$ & $5 \pm 1$ & 145 & $\ldots$ & $19 \% \pm 5 \%(24 / 127)$ & K0-M4 & Carpenter et al. (2006) \\
\hline NGC 2362 & $5 \pm 1$ & 1480 & $\ldots$ & $19 \% \pm 5 \%(38 / 195)$ & $\mathrm{K} 1-\mathrm{M} 4$ & Dahm \& Hillenbrand (2007) \\
\hline$\gamma \mathrm{Vel}^{\mathrm{a}}$ & $5 \pm 1.5$ & 350 & $\ldots$ & $6 \% \pm 2 \%(29 / 579)$ & K5 & Hernández et al. (2008) \\
\hline$\eta$ Cha & $7 \pm 1$ & 97 & $\ldots$ & $50 \% \pm 5 \%(8 / 15)$ & K4-M4 & Sicilia-Aguilar et al. (2009) \\
\hline NGC $7160^{\mathrm{a}}$ & $10 \pm 2$ & 900 & $\cdots$ & $4 \% \pm 2 \%(2 / 55)$ & K0-M1 & Sicilia-Aguilar et al. (2006) \\
\hline
\end{tabular}

Notes.

${ }^{a}$ High-mass cluster hosting $1 / 2$ OB stars.

b High-mass cluster hosting $>5$ OB stars.

c See Section 6.3 for the disk fraction.

members with masses $\gtrsim 1.0 M_{\odot}$. The IR/X-ray surveys are complete down to $1 M_{\odot}$ or $1.2 M_{\odot}$ computed with the $4 \mathrm{Myr}$ or $5 \mathrm{Myr}$ isochrones, respectively. The age of the cluster is determined via the position of the Class III stars (stars with no disks) on the [3.6]-[3.6]-[4.5] CMD. For IC 1795 we determine an age of 3-5 Myr.

The spatial distribution of the cluster was found to be asymmetric around the cluster center and no mass segregation is present.

The disk fraction was analyzed as a function of the distance from the cluster center as well as a function of the stellar mass. The objects with disks represent the $50 \%$ of the total source number. No spatial dependence of the disk frequency was found.

Using the [3.6] IRAC magnitude as a tracer for the stellar mass we find that the disk fraction is $\sim 20 \%$ for masses $>2 M_{\odot}$ and $\sim 50 \%$ for masses $<2 M_{\odot}$. We confirm that the dissipation of disks around high-mass stars $\left(>2 M_{\odot}\right)$ is faster compared to the dissipation around stars of $1-2 M_{\odot}$.

We found no variation in the disk fraction within $0.6 \mathrm{pc}$ of the O-type stars in the association.

Measurements of the disk fractions in low-mass and highmass environments have been collected from the literature. We found that, in general, the disk dissipation timescale is comparable in high-mass and low-mass clusters.

We are grateful to the Spitzer Science Center for their support during the data reduction. We acknowledge Leisa Townsley for supplying the Chandra/ACIS source list for W3 and thank Patrick Broos for assistance with matching those X-ray sources to the IRAC sources. We acknowledge G. Rodighiero for the helpful discussion about the extragalactic contamination in the mid-infrared. This publication makes use of data products from the Two Micron All Sky Survey, which is a joint project of the University of Massachusetts and the Infrared Processing and Analysis Center/California Institute of Technology, funded by the National Aeronautics and Space Administration and the National Science Foundation. E.D.F. was supported by Chandra grant SV4-74018, NASA grant NNX09AC74G, and NSF grant AST-0908038. A.S.A. is supported by the Deutsche Forschungsgemeinschaft (DFG) grant number SI 1486/1-1.

\section{APPENDIX A}

\section{EVOLUTIONARY MODEL}

The isochrones adopted for analysis have been computed using the FRANEC evolutionary code. We briefly describe here the physical inputs in the code. For detailed explanation the reader can refer to Chieffi \& Straniero (1989) and Degl'Innocenti et al. (2008). The opacity tables are from Ferguson et al. (2005) for $\log T[K]<4.5$ and from Iglesias $\&$ Rogers (1996) for higher temperatures. The equation of state (EOS) is described in Rogers et al. (1996). Both opacity tables and EOS are calculated for a heavy elements mixture equal to the solar mixture of Asplund et al. (2005). Our models are completely self-consistent, with a unique solar chemical composition, $(Y, Z)=(0.27,0.02)$. The value of the mixing length parameter adopted in the models is $\alpha_{\mathrm{MLT}}=1.6$.

Transformations from the theoretical $\left(\log \left(T_{\mathrm{eff}}[K]\right)\right.$, $\left.\log \left(L / L_{\odot}\right)\right)$ to the observational planes have been performed via synthetic photometry. In particular we have computed the isochrones in the $2 \mathrm{MASS}$ and $V I_{\text {Bessell }}$ photometric systems and for the first time in the IRAC photometric system. The optical and near-IR isochrones are consistent with the PMS isochrones computed by Siess et al. (2000). The filter throughputs and zero points of the IRAC photometric system are defined in Reach et al. (2005). We used stellar spectra generated with both the ATLAS9 (see Castelli \& Kurucz 2003) and PHOENIX (Brott \& Hauschildt 2005) model atmospheres codes.

The ATLAS9 grid of models is limited to temperatures higher than $3500 \mathrm{~K}$; corresponding isochrones then have a lower cut in mass at values of $0.36 M_{\odot}$ and $0.38 M_{\odot}$ for 3 and $5 \mathrm{Myr}$, respectively. The PHOENIX grid can cover a region of lower 

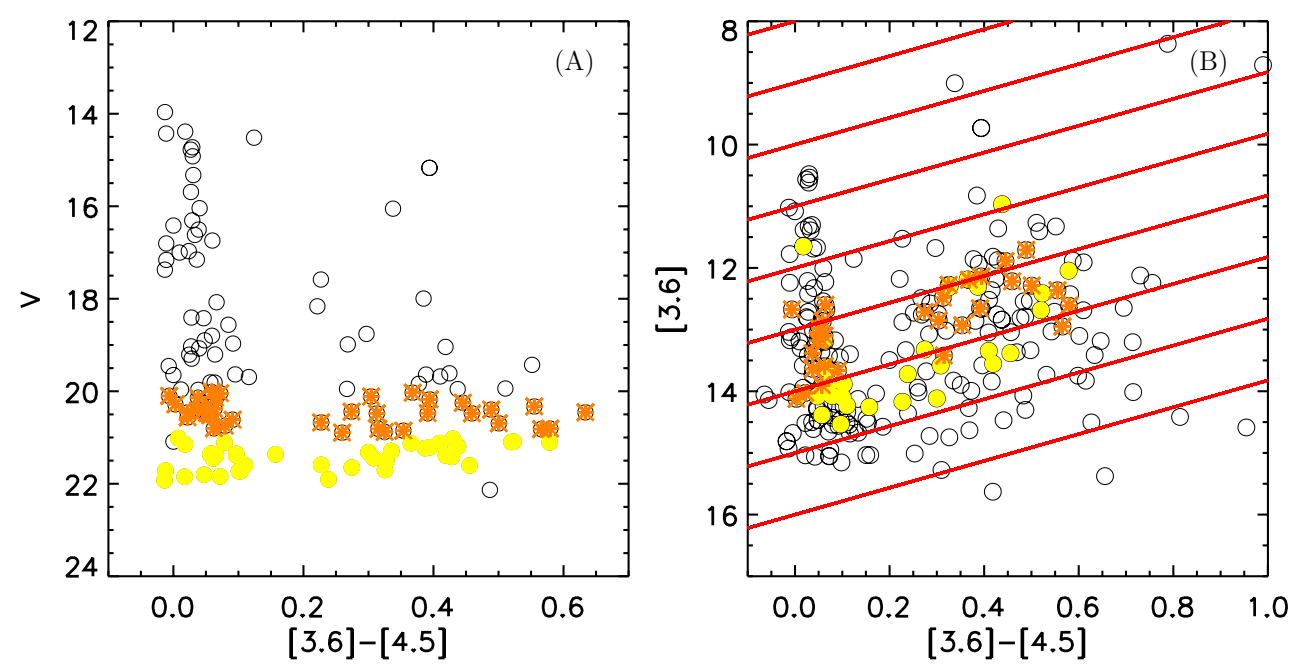

Figure 14. (A) [[3.6]-[4.5], V] CMD. Highlighted with different symbols are the stars in two consecutive horizontal slices with $V$ between 20 and 21 and with $V$ between 21 and 22. (B) [[3.6]-[4.5],[3.6]] CMD. Highlighted are the same sources as in panel (A). The derived lines used to compute the disk fraction are overplotted. (A color version of this figure is available in the online journal.)

temperatures $(T>2000 \mathrm{~K})$ but has an upper limit in temperature of $10,000 \mathrm{~K}$. This corresponds to $2.96 M_{\odot}$ and $2.56 M_{\odot}$ for 3 and $5 \mathrm{Myr}$, respectively.

\section{A.1. Mid-infrared Isochrones}

In the mid-infrared (MIR) CMDs, the part of the isochrone for intermediate-to-high-mass stars that have already reached the main sequence (MS) is almost vertical. Lower mass stars are still in the PMS phase. The PMS objects are cooler and with lower surface gravities; their spectra at the MIR wavelengths are no longer described by an (approximate) black body exponential tail, as in the MS phase. Due to the presence of spectral features the PMS isochrones show tendency toward redder colors.

PMS stars have larger radii when they are at the top of their Hayashi track, and the radii become smaller as the stars evolve and contract. In the totally convective phase the effective temperature changes little, leaving the observed color almost unchanged, even though the change in surface gravity slightly affects the shape of the emerging spectrum. But with decreasing age, the change in radius heavily affects the total luminosity. For this reason mid-infrared PMS isochrones are no longer age-degenerate as they are in the MS phase. On the contrary different PMS isochrones are well separated in the MIR CMDs and can be used for age-dating the cluster, or, at least, setting some constraint on its age. In particular, the transition phase between PMS and MS is characterized by a hook-shaped bending in the isochrone. As the age increases, the hook and the red PMS branch move toward larger magnitudes (lower luminosities). When an independent estimate of the distance and the extinction is available, as in our case, the Main-Sequence Turn-On (MSTON) can be used as upper limit for the age of a cluster. After placing an isochrone in the CMD, if no star is observed below the MSTON, at color equal zero, then the cluster must be younger than the given isochrone.

For this reason the position of the MSTON is used to infer the age of the cluster.

\section{APPENDIX B}

\section{DISK FRACTION IN THE [[3.6],[3.6]-[4.5]] CMD}

Figure 14(A) shows the [[3.6]-[4.5], V] CMD. In this diagram the presence of the CS disk causes the object to move horizontally toward red colors. This is because the disk emission affects only slightly the $V$ magnitude (e.g., through optical veiling produced by mass accretion).

In the [[3.6]-[4.5],[3.6]] CMD shown in Figure 14(B), disk emission also affects the stellar luminosity ([3.6] mag). As a consequence the presence of the disk will move the object toward red colors obliquely.

To properly compute the disk fraction as a function of the [3.6] mag (and hence stellar mass) it is necessary to take into account this effect. We calculate the isomass in the [3.6] mag in the [[3.6]-[4.5],[3.6]] CMD. This was computed in the following way: we select all stars in a series of horizontal slices in the [[3.6]-[4.5], $V] \mathrm{CMD}$ (central $V=20.5 ; 21.5 \mathrm{mag}$ and bin $=0.5 \mathrm{mag}$ ). We look for the position of the same objects (highlighted with the same symbols) in the [[3.6],[3.6]-[4.5]] CMD. We extrapolate the median inclination of the position of these sources in this CMD for each magnitude bin. Finally, we computed the disk fraction in a series of bins counting the sources in the oblique slices.

\section{APPENDIX C}

\section{RELATION BETWEEN 3.6 $\mu \mathrm{m}$ IRAC MAGNITUDE AND STELLAR MASS}

Due to the spread of the sources along the isochrone, we assign the most probable mass by taking into account the photometric uncertainty via a maximum-likelihood method. We define the likelihood function for the $i$ th observed star as

$$
\mathcal{L}^{i}\left(m_{j}\right)=\frac{1}{2 \pi \sigma_{3.6}^{i} \sigma_{4.5}^{i}} \times \exp \left(-\chi^{2} / 2\right),
$$

where

$$
\chi^{2}=\left(\frac{[3.6]_{o b s}^{i}-[3.6]_{t h}^{j}}{\sigma_{3.6}^{i}}\right)^{2}+\left(\frac{[4.5]_{o b s}^{i}-[4.5]_{t h}^{j}}{\sigma_{4.5}^{i}}\right)^{2},
$$

here the $j$ index runs on the masses along the isochrone and $\sigma^{i}$ are the photometric uncertainties for the $i$ th star and subscripts $o b s$ and $t h$ stand for observed and predicted quantities, respectively. Since we use the magnitude-magnitude space the uncertainties can be considered uncorrelated and the $\chi^{2}$ assumes the form of 
Equation (C2). We assign to the $i$ th data point the mass value $m_{j *}$ for which $\mathcal{L}\left(m_{j}\right)$ has its maximum.

\section{REFERENCES}

Adams, F. C., Hollenbach, D., Laughlin, G., \& Gorti, U. 2004, ApJ, 611, 360 Allen, L. E., et al. 2004, ApJS, 154, 363

Asplund, M., Grevesse, N., \& Sauval, A. J. 2005, in ASP Conf. Ser. 336, Cosmic Abundances as Records of Stellar Evolution and Nucleosynthesis, ed. T. G. Barnes, III \& F. N. Bash (San Franciso, CA: ASP), 25

Bally, J., Sutherland, R. S., Devine, D., \& Johnstone, D. 1998, AJ, 116, 293

Balog, Z., Muzerolle, J., Rieke, G. H., Su, K. Y. L., Young, E. T., \& Megeath, S. T. 2007, ApJ, 660, 1532

Binney, J., \& Tremaine, S. 1987, Galactic Dynamics (Princeton, NJ: Princeton Univ. Press)

Bonatto, C., Santos, J. F. C., Jr., \& Bica, E. 2006, A\&A, 445, 567

Bouwman, J., Lawson, W. A., Dominik, C., Feigelson, E. D., Henning, T., Tielens, A. G. G. M., \& Waters, L. B. F. M. 2006, ApJ, 653, L57

Broos, P. S., Feigelson, E. D., Townsley, L. K., Getman, K. V., Wang, J., Garmire, G. P., Jiang, Z., \& Tsuboi, Y. 2007, ApJS, 169, 353

Broos, P. S., Townsley, L. K., Feigelson, E. D., Getman, K. V., Bauer, F. E., \& Garmire, G. P. 2010, ApJ, 714, 1582

Brott, I., \& Hauschildt, P. H. 2005, in Proc. Gaia Symposium, The ThreeDimensional Universe with Gaia, ed. C. Turon, K. S. O’Flaherty, \& M. A. C. Perryman (ESA Special Publication, Vol. 576), 565

Cardelli, J. A., Clayton, G. C., \& Mathis, J. S. 1989, ApJ, 345, 245

Carpenter, J. M., Mamajek, E. E., Hillenbrand, L. A., \& Meyer, M. R. 2006, ApJ, 651, L49

Castelli, F., \& Kurucz, R. L. 2003, in IAU Symp. 210, Modeling of Stellar Atmospheres, ed. N. Piskunov, W. W. Weiss, \& D. F. Gray (Cambridge: Cambridge Univ. Press), 20

Chieffi, A., \& Straniero, O. 1989, ApJS, 71, 47

Clarke, C. J. 2007, MNRAS, 376, 1350

Currie, T., et al. 2007, ApJ, 659, 599

Dahm, S. E., \& Hillenbrand, L. A. 2007, AJ, 133, 2072

Damiani, F., Prisinzano, L., Micela, G., \& Sciortino, S. 2006, A\&A, 459, 477

Degl'Innocenti, S., Prada Moroni, P. G., Marconi, M., \& Ruoppo, A. 2008, Ap\&SS, 316, 25

Eisner, J. A., Plambeck, R. L., Carpenter, J. M., Corder, S. A., Qi, C., \& Wilner, D. 2008, ApJ, 683, 304

Evans, N. J., et al. 2009, ApJS, 181, 321

Fang, M., van Boekel, R., Wang, W., Carmona, A., Sicilia-Aguilar, A., \& Henning, T. 2009, A\&A, 504, 461

Fazio, G. G., et al. 2004, ApJS, 154, 10

Fedele, D., van den Ancker, M. E., Henning, T., Jayawardhana, R., \& Oliveira, J. M. 2010, A\&A, 510, A72

Feigelson, E., Townsley, L., Güdel, M., \& Stassun, K. 2007, in Protostars and Planets V, ed. B. Reipurth, D. Jewitt, \& K. Keil (Tucson, AZ: Univ. Arizona Press), 313

Feigelson, E. D., Martin, A. L., McNeill, C. J., Broos, P. S., \& Garmire, G. P. 2009, AJ, 138, 227

Feigelson, E. D., \& Townsley, L. K. 2008, ApJ, 673, 354

Ferguson, J. W., Alexander, D. R., Allard, F., Barman, T., Bodnarik, J. G., Hauschildt, P. H., Heffner-Wong, A., \& Tamanai, A. 2005, ApJ, 623, 585

Flaherty, K. M., \& Muzerolle, J. 2008, AJ, 135, 966

Franceschini, A., et al. 2006, A\&A, 453, 397

Getman, K. V., Feigelson, E. D., Luhman, K. L., Sicilia-Aguilar, A., Wang, J., \& Garmire, G. P. 2009, ApJ, 699, 1454

Getman, K. V., Feigelson, E. D., Townsley, L., Broos, P., Garmire, G., \& Tsujimoto, M. 2006, ApJS, 163, 306

Gorti, U., Dullemond, C. P., \& Hollenbach, D. 2009, ApJ, 705, 1237

Guarcello, M. G., Micela, G., Damiani, F., Peres, G., Prisinzano, L., \& Sciortino, S. 2009, A\&A, 496, 453

Guarcello, M. G., Prisinzano, L., Micela, G., Damiani, F., Peres, G., \& Sciortino, S. 2007, A\&A, 462, 245

Gutermuth, R. A., Megeath, S. T., Muzerolle, J., Allen, L. E., Pipher, J. L., Myers, P. C., \& Fazio, G. G. 2004, ApJS, 154, 374

Gutermuth, R. A., Megeath, S. T., Myers, P. C., Allen, L. E., Pipher, J. L., \& Fazio, G. G. 2009, ApJS, 184, 18

Gutermuth, R. A., et al. 2008, ApJ, 674, 336

Hachisuka, K., et al. 2006, ApJ, 645, 337

Hartmann, L., Megeath, S. T., Allen, L., Luhman, K., Calvet, N., D’Alessio, P., Franco-Hernandez, R., \& Fazio, G. 2005, ApJ, 629, 881
Hatchell, J., Fuller, G. A., Richer, J. S., Harries, T. J., \& Ladd, E. F. 2007, A\&A, 468, 1009

Henning, T., \& Meeus, G. 2009, arXiv:0911.1010

Hernández, J., Hartmann, L., Calvet, N., Jeffries, R. D., Gutermuth, R., Muzerolle, J., \& Stauffer, J. 2008, ApJ, 686, 1195

Hernández, J., et al. 2007, ApJ, 662, 1067

Hillenbrand, L. A. 1997, AJ, 113, 1733

Hillenbrand, L. A., Strom, S. E., Calvet, N., Merrill, K. M., Gatley, I., Makidon, R. B., Meyer, M. R., \& Skrutskie, M. F. 1998, AJ, 116, 1816

Iglesias, C. A., \& Rogers, F. J. 1996, ApJ, 464, 943

Jeffries, R. D. 2007, MNRAS, 376, 1109

Jeffries, R. D., Oliveira, J. M., Naylor, T., Mayne, N. J., \& Littlefair, S. P. 2007, MNRAS, 376, 580

Johnstone, D., Hollenbach, D., \& Bally, J. 1998, ApJ, 499, 758

Kennedy, G. M., \& Kenyon, S. J. 2009, ApJ, 695, 1210

Kroupa, P. 2002, Science, 295, 82

Lada, C. J., et al. 2006, AJ, 131, 1574

Luhman, K. L., Allen, P. R., Espaillat, C., Hartmann, L., \& Calvet, N. 2010, ApJS, 186, 111

Luhman, K. L., et al. 2008, ApJ, 675, 1375

Lyo, A., Lawson, W. A., Mamajek, E. E., Feigelson, E. D., Sung, E., \& Crause, L. A. 2003, MNRAS, 338, 616

Mamajek, E. E., Meyer, M. R., Hinz, P. M., Hoffmann, W. F., Cohen, M., \& Hora, J. L. 2004, ApJ, 612, 496

Mann, R. K., \& Williams, J. P. 2009, ApJ, 694, L36

Mathys, G. 1989, A\&AS, 81, 237

McCullough, P. R., Fugate, R. Q., Christou, J. C., Ellerbroek, B. L., Higgins, C. H., Spinhirne, J. M., Cleis, R. A., \& Moroney, J. F. 1995, ApJ, 438, 394

Meeus, G., et al. 2009, A\&A, 497, 379

Megeath, S. T., et al. 2004, ApJS, 154, 367

Mentuch, E., Brandeker, A., van Kerkwijk, M. H., Jayawardhana, R., \& Hauschildt, P. H. 2008, ApJ, 689, 1127

Mercer, E. P., Miller, J. M., Calvet, N., Hartmann, L., Hernandez, J., SiciliaAguilar, A., \& Gutermuth, R. 2009, AJ, 138, 7

O'dell, C. R., \& Wong, K. 1996, AJ, 111, 846

Oey, M. S., Watson, A. M., Kern, K., \& Walth, G. L. 2005, AJ, 129, 393

Olczak, C., Pfalzner, S., \& Spurzem, R. 2006, ApJ, 642, 1140

Oliveira, J. M., Jeffries, R. D., van Loon, J. T., Littlefair, S. P., \& Naylor, T. 2005, MNRAS, 358, L21

Oliveira, J. M., Jeffries, R. D., van Loon, J. T., \& Rushton, M. T. 2006, MNRAS, 369,272

Pascucci, I., Apai, D., Luhman, K., Henning, T., Bouwman, J., Meyer, M. R., Lahuis, F., \& Natta, A. 2009, ApJ, 696, 143

R Development Core Team 2009, R: A Language and Environment for Statistical Computing (Vienna, Austria: R Foundation for Statistical Computing), 409

Reach, W. T., et al. 2005, PASP, 117, 978

Richards, G. T., et al. 2009, AJ, 137, 3884

Rogers, F. J., Swenson, F. J., \& Iglesias, C. A. 1996, ApJ, 456, 902

Ruch, G. T., Jones, T. J., Woodward, C. E., Polomski, E. F., Gehrz, R. D., \& Megeath, S. T. 2007, ApJ, 654, 338

Scally, A., \& Clarke, C. 2001, MNRAS, 325, 449

Sicilia-Aguilar, A., Hartmann, L. W., Hernández, J., Briceño, C., \& Calvet, N. 2005, AJ, 130, 188

Sicilia-Aguilar, A., Henning, T., \& Hartmann, L. W. 2010, ApJ, 710, 597

Sicilia-Aguilar, A., Henning, T., Juhász, A., Bouwman, J., Garmire, G., \& Garmire, A. 2008, ApJ, 687, 1145

Sicilia-Aguilar, A., et al. 2006, ApJ, 638, 897

Sicilia-Aguilar, A., et al. 2009, ApJ, 701, 1188

Siess, L., Dufour, E., \& Forestini, M. 2000, A\&A, 358, 593

Skrutskie, M. F., et al. 2006, AJ, 131, 1163

Stelzer, B., \& Scholz, A. 2009, A\&A, 507, 227

Telleschi, A., Güdel, M., Briggs, K. R., Audard, M., \& Palla, F. 2007, A\&A, 468, 425

Wang, J., Feigelson, E. D., Townsley, L. K., Román-Zúñiga, C. G., Lada, E., \& Garmire, G. 2009, ApJ, 696, 47

Wang, J., Townsley, L. K., Feigelson, E. D., Broos, P. S., Getman, K. V., RománZúñiga, C. G., \& Lada, E. 2008, ApJ, 675, 464

Weisskopf, M. C., Brinkman, B., Canizares, C., Garmire, G., Murray, S., \& Van Speybroeck, L. P. 2002, PASP, 114, 1

Werner, M. W., et al. 2004, ApJS, 154, 1

Winston, E., et al. 2007, ApJ, 669, 493

Xu, Y., Reid, M. J., Zheng, X. W., \& Menten, K. M. 2006, Science, 311, 54 